\title{
Non-Abelian Alice strings in two-flavor dense QCD
}

\author{
Yuki Fujimoto, ${ }^{1, *}$ and Muneto Nitta ${ }^{2, \dagger}$ \\ ${ }^{1}$ Department of Physics, The University of Tokyo, 7-3-1 Hongo, Bunkyo-ku, Tokyo 113-0033, Japan \\ ${ }^{2}$ Department of Physics \& Research and Education Center for Natural Sciences, \\ Keio University, Hiyoshi 4-1-1, Yokohama, Kanagawa 223-8521, Japan
}

(Received 26 November 2020; accepted 9 February 2021; published 1 March 2021)

\begin{abstract}
Quark-hadron continuity with two-flavor quarks that was proposed recently connects hadronic matter with neutron ${ }^{3} P_{2}$ superfluidity and two-flavor dense quark matter. This two-flavor dense quark phase consists of the coexistence of the 2SC condensates and the $P$-wave diquark condensates of $d$-quarks, which gives rise to color superconductivity as well as superfluidity. We classify vortices in this phase. The most stable vortices are what we call the non-Abelian Alice strings, which are superfluid vortices with nonAbelian color magnetic fluxes therein, exhibiting so-called topological obstruction, or a non-Abelian generalization of the Alice property. We show that a single Abelian superfluid vortex is unstable against decay into three non-Abelian Alice strings. We discover that a non-Abelian Alice string carries orientational moduli of the real projective space $\mathbb{R} P^{2}$ corresponding to the color flux therein in the presence of the $P$-wave condensates alone. We calculate Aharanov-Bohm (AB) phases around the nonAbelian Alice string, and find that the 2SC condensates and string's orientational moduli must be aligned with each other because of single-valuedness of the $\mathrm{AB}$ phases of the $2 \mathrm{SC}$ condensates.
\end{abstract}

DOI: 10.1103/PhysRevD.103.054002

\section{INTRODUCTION}

Color superconductor is the ground state of the cold QCD matter at densities much higher than that of saturated nuclei $n_{0}=0.16 \mathrm{fm}^{-3}$; the only known circumstance where we might find such kind of matter is in the core of neutron stars [1]. Various phases are known for color superconductivity such as color-flavor locked (CFL) phase [2] in three-flavor symmetric matter and 2-flavor superconducting $(2 \mathrm{SC})$ phase $[3,4]$ in two-flavor symmetric matter.

Quantum vortices or flux tubes arise in color-superconducting quark matter [5]. In the CFL phase, topologically stable superfluid vortex comes about owing to the nontrivial first homotopy group $\pi_{1}\left[\mathrm{U}(1)_{\mathrm{B}}\right]=\mathbb{Z}[6,7]$. It is related with the broken $\mathrm{U}(1)_{\mathrm{B}}$ symmetry in the CFL phase, which is possible as the Vafa-Witten theorem does not apply at finite density [8]. The minimal stable configuration in the CFL phase is known to be the non-Abelian semisuperfluid vortices carrying color magnetic flux and only $1 / 3$ of the circulation of the Abelian superfluid vortices

\footnotetext{
fujimoto@nt.phys.s.u-tokyo.ac.jp

nitta@phys-h.keio.ac.jp
}

Published by the American Physical Society under the terms of the Creative Commons Attribution 4.0 International license. Further distribution of this work must maintain attribution to the author(s) and the published article's title, journal citation, and DOI. Funded by SCOAP.
[5,9-12]. Recently, in the context of the quark-hadron continuity [13-18] - the concept that color superconductor and hadronic superfluid are continuously connected building upon the identical symmetry breaking patterns and low-lying excitations in the both phases-connection of the CFL vortices with the hadronic ones has been discussed [19-24].

In contrast, in the $2 \mathrm{SC}$ phase, it has been thought that topologically stable vortices do not appear since the $\mathrm{U}(1)_{\mathrm{B}}$ stays intact resulting in a trivial first homotopy group, unlike in the CFL phase [25]. The recent insights from the neutron star observations, however, lead to the novel phase of color superconductivity in two-flavor matter with the broken $\mathrm{U}(1)_{\mathrm{B}}$ symmetry [26]. This phase is called the $2 \mathrm{SC}+\langle d d\rangle$ phase. Here, we comment on how the $2 \mathrm{SC}+$ $\langle d d\rangle$ phase comes into play. Our current understanding of the cold high-density QCD matter relies on the quantity called equation of state (EoS), which is a pressure function $P\left(n_{\mathrm{B}}\right)$ of the baryon number density $n_{\mathrm{B}}$ at $T=0$. At densities around $n_{\mathrm{B}} \sim n_{0}$, description by nucleon degrees of freedom works well and the matter is dominated by neutrons. Meanwhile, at higher densities, the matter is well described in terms of quark degrees of freedom. According to the current model-independent analysis of the neutron star EoS, there might be a possibility that a substantial quark core exists inside a heavy neutron star [27]. The sizable quark core inside the neutron star can be realized by a smooth crossover from the hadronic matter to the quark matter in the EoS [28-30]. This crossover 


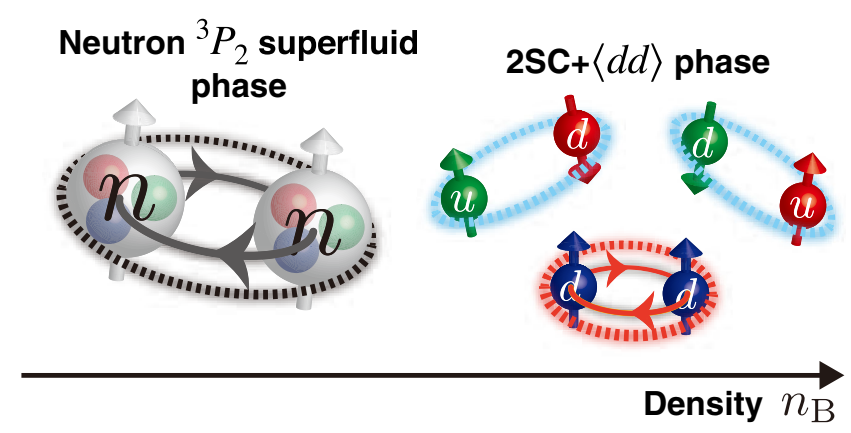

FIG. 1. Illustration of the quark-hadron continuity from the neutron ${ }^{3} P_{2}$ superfluid phase to the $2 \mathrm{SC}+\langle d d\rangle$ phase.

construction satisfies the stringent constraints put by observations, e.g., the two-solar-mass conditions [31]. The rationale behind the crossover construction is the concept of quark-hadron continuity. The quark-hadron continuity has been previously formulated in the ideal $\mathrm{SU}(3)_{\mathrm{F}}$ flavor symmetric setup [13-17]. At $n_{\mathrm{B}} \sim n_{0}$, the matter is dominated by neutrons, which are composed of two-flavor $u$ and $d$ valence quarks, not by hyperons that contains $s$ quark. Moreover, neutrons are known to be paired up in the ${ }^{3} P_{2}$ channel and show the superfluidity [32-36] (see also Refs. [37-39] and references therein for recent studies). To fit with this natural ground state at $n_{\mathrm{B}} \sim n_{0}$, we are lead to the more realistic picture of quarkhadron continuity in the $\mathrm{SU}(2)_{\mathrm{F}}$ flavor symmetric setupthe neutron ${ }^{3} P_{2}$ superfluid is continuously connected to the two-flavor quark matter-in accordance with the abovementioned crossover EoS. As a consequence, the new condensate $\left\langle\hat{d}^{T} \mathcal{C} \gamma^{i} \nabla^{j} \hat{d}\right\rangle$, which is a diquark of $d$-quarks paired in the $P$-wave channel, appears in addition to the conventional two-flavor $2 \mathrm{SC}$ condensate. We will use the shorthand notation $\langle d d\rangle$ instead of $\left\langle\hat{d}^{T} \mathcal{C} \gamma^{i} \nabla^{j} \hat{d}\right\rangle$ throughout this work unless otherwise specified. The coexistence of the 2SC and $\langle d d\rangle$ condensates in the 2SC $+\langle d d\rangle$ phase ensures the continuity to hold as illustrated in Fig. 1.

In this work, on the basis of this $2 \mathrm{SC}+\langle d d\rangle$ phase picture, we study vortices arising in the two-flavor dense quark matter for the first time. We describe that the most stable vortices in the $\langle d d\rangle$ phase are non-Abelian vortices, which support $1 / 3$ fractional windings in $\mathrm{U}(1)_{\mathrm{B}}$ as well as the color-magnetic fluxes similar to those in the CFL phase. These vortices also exhibit the unique features akin to the so-called Alice strings [40-50], thus we named them as "non-Abelian Alice strings". This feature is what is called topological obstruction, implying that some unbroken generators in the bulk are not globally defined around the string. We also show that a single Abelian $\mathrm{U}(1)_{\mathrm{B}}$ vortex is unstable against a decay into triple of non-Abelian Alice strings, as the case of those in the CFL phase [10,20,51,52]. We then show that a non-Abelian Alice string carries orientational moduli, or collective coordinates, of the real projective space $\mathbb{R} P^{2}$ corresponding to the color flux therein in the presence of the $\langle d d\rangle$ condensates alone, in contrast to those in the CFL phase carrying moduli of the complex projective space $\mathbb{C} P^{2}[5,10,53,54]$. We show that quasi-quarks exhibit nontrivial (generalized) AharanovBohm (AB) phases around the non-Abelian Alice string, and then find the "bulk-soliton moduli locking" phenomenon, i.e., when the $2 \mathrm{SC}$ condensates develop VEVs, they must be aligned to string's orientational moduli because of single-valuedness of the $\mathrm{AB}$ phases of them.

This paper is organized as follows. In Sec. II, we will further review the structure of the $2 \mathrm{SC}+\langle d d\rangle$ phase. We will consider the corresponding symmetry breaking patterns. In Sec. III, we will categorize vortices in the presence of $\langle d d\rangle$ diquark into three-types: Abelian superfluid vortices, color-magnetic flux tubes, and the non-Abelian Alice strings. In Sec. IV, we will discuss properties of the nonAbelian Alice string. We will also introduce the generalized $\mathrm{AB}$ phase around strings, and discuss that the Abelian vortices break up into three Alice strings based on the $A B$ phase. The most stable configuration among these turns out to be the non-Abelian Alice string. In Sec. V, we will point out that the moduli of vortex are aligned with the bulk quantity in the point of view of generalized $A B$ phase. We will make a comment on the consistency on the ordering of the diquark condensate formation in Sec. VI. In Sec. VII, we will finally summarize our results.

\section{TWO-FLAVOR DENSE QUARK MATTER}

In this section, after giving a summary of the two-flavor dense quark matter, especially the $2 \mathrm{SC}+\langle d d\rangle$ phase formulated in Refs. [26], we discuss symmetry breaking patterns in this phase.

\section{A. 2SC $+\langle d d\rangle$ phase from quark-hadron continuity}

The concept of quark-hadron continuity builds upon the so-called Fradkin-Shenker theorem [55-57] (see also [24] for recent model study on the validity of this theorem). This folk theorem states that within a gauge-Higgs theory, we cannot distinguish between the confinement and Higgs phases with any local order parameters as long as global symmetries in these phases are the same. We apply this theorem to the twoflavor dense QCD, where confinement and Higgs phases correspond to the hadronic and color-superconducting quark phases, respectively. Here, we take a superfluid operator as an example of a local order parameter with which we cannot distinguish these two phases.

The hadronic phase in this case is a neutron ${ }^{3} P_{2}$ superfluid, for which the order parameter operator is given by $[32,33,36]$

$$
\hat{A}^{i j}=\hat{n}^{T} \mathcal{C} \gamma^{i} \nabla^{j} \hat{n}
$$

where $\hat{n}$ denotes a neutron field operator, $\mathcal{C}$ is the charge conjugation operator, and indices $(i, j, \ldots)$ denote spatial 
coordinates. In this paring, the matrices $\gamma^{i}$ and spatial derivatives $\nabla^{j}$ account for spin and angular momentum contributions in the ${ }^{3} P_{2}$ pairing, respectively. We further assume that neutrons made out of $u$ and $d$-quarks can be described as a quark-diquark system $\hat{n}=\epsilon^{\alpha \beta \gamma}\left(\hat{u}_{\alpha}^{T} \mathcal{C} \gamma^{5} \hat{d}_{\beta}\right) \hat{d}_{\gamma}$ with the Greek letters $(\alpha, \beta, \ldots)$ being the color indices. With this in mind, $\hat{A}^{i j}$ can be rearranged into three diquarks as

$$
\begin{aligned}
\hat{A}^{i j} & \propto \epsilon^{\alpha \beta \gamma} \epsilon^{\alpha^{\prime} \beta^{\prime} \gamma^{\prime}}\left(\hat{u}_{\alpha}^{T} \mathcal{C} \gamma^{5} \hat{d}_{\beta}\right)\left(\hat{u}_{\alpha^{\prime}}^{T} \mathcal{C} \gamma^{5} \hat{d}_{\beta^{\prime}}\right)\left(\hat{d}_{\gamma}^{T} \mathcal{C} \gamma^{i} \nabla^{j} \hat{d}_{\gamma^{\prime}}\right) \\
& =\left(\hat{\Phi}_{2 \mathrm{SC}}\right)^{\gamma}\left(\hat{\Phi}_{2 \mathrm{SC}}\right)^{\gamma^{\prime}}\left(\hat{\Phi}_{d d}\right)_{\gamma \gamma^{\prime}}^{i j}
\end{aligned}
$$

with $\hat{\Phi}_{2 S C}$ and $\hat{\Phi}_{d d}$ being the diquark operators defined as

$$
\begin{gathered}
\left(\hat{\Phi}_{2 \mathrm{SC}}\right)^{\alpha} \equiv \epsilon^{\alpha \beta \gamma} \hat{u}_{\beta}^{T} \mathcal{C} \gamma^{5} \hat{d}_{\gamma}, \\
\left(\hat{\Phi}_{d d}\right)_{\alpha \beta}^{i j} \equiv \hat{d}_{\alpha}^{T} \mathcal{C} \gamma^{i} \nabla^{j} \hat{d}_{\beta} .
\end{gathered}
$$

One can take the expectation value of $\hat{A}^{i j}$ in the hadronic phase, which reads

$$
\left\langle\hat{A}^{i j}\right\rangle=\left\langle\hat{n}^{T} \mathcal{C} \gamma^{i} \nabla^{j} \hat{n}\right\rangle
$$

This condensate accounts for the ${ }^{3} P_{2}$ superfluidity of the neutron matter.

By contrast, one can also take the expectation value of $\hat{A}^{i j}$ in the quark phase under the mean field approximation in light of Eq. (2):

$$
\left\langle\hat{A}^{i j}\right\rangle \simeq\left(\Phi_{2 \mathrm{SC}}\right)^{\alpha}\left(\Phi_{2 \mathrm{SC}}\right)^{\beta}\left(\Phi_{d d}\right)_{\alpha \beta}^{i j},
$$

where we have defined

$$
\begin{gathered}
\Phi_{2 \mathrm{SC}} \equiv\left\langle\hat{\Phi}_{2 \mathrm{SC}}\right\rangle, \\
\Phi_{d d} \equiv\left\langle\hat{\Phi}_{d d}\right\rangle .
\end{gathered}
$$

Note that quantities without hat symbols denote condensates while those with hat symbols are operators. The two condensates $\Phi_{2 \mathrm{SC}}$ and $\Phi_{d d}$ at the mean field level in Eq. (6) account for the color superconductivity of the quark matter: $\Phi_{2 S C}$ is the so-called 2SC condensate, while the novel feature here is represented by $\Phi_{d d}$, which is the diquark condensate of $d$-quarks in the ${ }^{3} P_{2}$ channel. We understand the indices $\alpha, \beta$ of $\left(\Phi_{d d}\right)_{\alpha \beta}$ are in 6 representation of color as they should be symmetric. Qualitatively speaking, this ${ }^{3} P_{2}$ pairing arises owing to the short range repulsion in the color 6 channel of one-gluon exchange (OGE), which disfavors the $S$-wave pairing, and the selective attraction in the $J=2$ channel, which arises from the spin-orbit dependent part of the Fermi-Breit reduced interaction of OGE. We call this coexistence phase of $\Phi_{2 S C}$ and $\Phi_{d d}$ as the $2 \mathrm{SC}+\langle d d\rangle$ phase.
As mentioned above, $\left\langle A^{i j}\right\rangle$ is always nonzero in both phases, so the local order parameter indeed cannot distinguish these two phases. It leads us to the continuity. For more details, see Ref. [26].

\section{B. Symmetry of the color-superconducting phase}

We consider the symmetry group of QCD as $G_{\mathrm{QCD}}=$ $\mathrm{SU}(3)_{\mathrm{C}} \times \mathrm{U}(1)_{\mathrm{B}}$, without paying attention to the chiral symmetry of QCD since it does not play an important role in discussion of this paper. Under the element $\left(U, e^{i \theta_{\mathrm{B}}}\right) \in$ $\mathrm{SU}(3)_{\mathrm{C}} \times \mathrm{U}(1)_{\mathrm{B}}$ acting on quark fields $q$, as a column vector belonging to the fundamental representation 3 of $\mathrm{SU}(3)_{\mathrm{C}}$, as

$$
q \rightarrow e^{i \theta_{\mathrm{B}}} U q
$$

where $U \in$ (the 3 representation of) $\mathrm{SU}(3)_{\mathrm{C}}$, the diquark condensates (7), (8) transforms as

$\Phi_{2 \mathrm{SC}} \rightarrow e^{2 i \theta_{\mathrm{B}}} U^{*} \Phi_{2 \mathrm{SC}}, \quad \Phi_{d d} \rightarrow e^{2 i \theta_{\mathrm{B}}} U \Phi_{d d} U^{T}$.

Here, the factor 2 in $\mathrm{U}(1)_{\mathrm{B}}$ is inserted to maintain the consistency with a single quark; we suppress the spatial indices $i, j$ of $\Phi_{d d}$ hereafter, where an appropriate tensor structure is implied [58].

Once the condensates $\Phi_{2 S C}$ and $\Phi_{d d}$ are nonzero, symmetry is spontaneously broken and superconductivity sets in. Here, we examine the subgroups that leave these condensates invariant and identify the corresponding symmetry breaking patterns. We consider two regimes in which each condensate is turned on sequentially instead of turning them on simultaneously. Namely, we firstly turn on $\Phi_{d d}$ and then turn on $\Phi_{2 S C}$ and choose the gauge so as to be consistent with each other, and vice versa. This can be summarized as

$$
\begin{aligned}
& \text { 1. } G_{\mathrm{QCD}} \stackrel{\Phi_{d d}}{\rightarrow} H_{d d} \stackrel{\Phi_{2 S C}}{\rightarrow} K_{2 \mathrm{SC}+d d} \\
& \text { 2. } G_{\mathrm{QCD}} \stackrel{\Phi_{2 \mathrm{SC}}}{\longrightarrow} \tilde{H}_{2 \mathrm{SC}} \stackrel{\Phi_{d d}}{\rightarrow} K_{2 \mathrm{SC}+d d}
\end{aligned}
$$

and also in Fig. 2. We will mainly focus on the first option

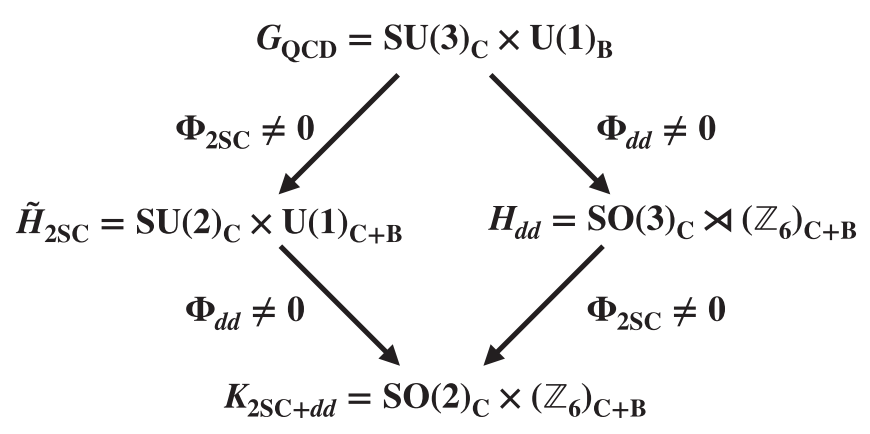

FIG. 2. Spontaneous symmetry breaking patterns in the most symmetric case. 
in this work. The second option is also mentioned to ensure the consistency.

\section{Symmetry breaking by the $\langle d d\rangle$ condensate}

First, let us turn to $G_{\mathrm{QCD}} \stackrel{\Phi_{d d}}{\rightarrow} H_{d d}$ part in Eq. (11). The matrix $\left(\Phi_{d d}\right)_{\alpha \beta}$ can be diagonalized by a gauge rotation, so that without loss of generality one can take

$$
\Phi_{d d}=\operatorname{diag}\left[\left(\Phi_{d d}\right)_{11},\left(\Phi_{d d}\right)_{22},\left(\Phi_{d d}\right)_{33}\right] .
$$

Here, for simplicity, we posit the most symmetric case that in the ground state $\left(\Phi_{d d}\right)_{11}=\left(\Phi_{d d}\right)_{22}=\left(\Phi_{d d}\right)_{33}=\Delta_{d d}$, thus

$$
\Phi_{d d}=\Delta_{d d} \mathbf{1}_{3}
$$

Then, the unbroken subgroup

$$
H_{d d}=\mathrm{SO}(3)_{\mathrm{C}} \rtimes\left(\mathbb{Z}_{6}\right)_{\mathrm{C}+\mathrm{B}}
$$

keeps $\Phi_{d d}=\Delta_{d d} \mathbf{1}_{3}$ invariant. The condition $U U^{T}=\mathbf{1}_{3}$, which comes from Eq. (10) taking $\theta_{\mathrm{B}}=0$, imposes $\mathrm{SO}(3)_{\mathrm{C}}$. The discrete group is defined by

$$
\left(\mathbb{Z}_{6}\right)_{\mathrm{C}+\mathrm{B}}:\left(X^{k}, \omega^{-2 k}\right) \in \mathrm{SU}(3)_{\mathrm{C}} \times \mathrm{U}(1)_{\mathrm{B}}
$$

with $k=0,1,2,3,4,5$ and $\omega \equiv e^{i \pi / 3}$ being the sixth root of unity;

$$
X \equiv \operatorname{diag}\left(\omega, \omega, \omega^{-2}\right)
$$

is generated by $T_{8} \propto \operatorname{diag}(1,1,-2)$ of the broken $\mathrm{SU}(3)_{\mathrm{C}}$ symmetry. We define the semidirect product $\rtimes_{\phi}$ in $H_{d d}$ by the group operation $\circ$ determined by the homomorphism $\phi$ :

$$
\begin{aligned}
\left(U, X^{k}\right) \circ\left(U^{\prime}, X^{k^{\prime}}\right) & =\left(U \phi_{X^{k}}\left(U^{\prime}\right), X^{k} X^{k^{\prime}}\right), \\
\phi_{X^{k}}\left(U^{\prime}\right) & \equiv X^{k} U^{\prime}\left(X^{k}\right)^{-1}
\end{aligned}
$$

where $\left(U^{(\prime)}, X^{k^{(\prime)}}\right) \in \mathrm{SO}(3)_{\mathrm{C}} \times\left(\mathbb{Z}_{6}\right)_{\mathrm{C}+\mathrm{B}} . \quad \phi_{X^{k}}$ is trivial when $k$ is even, but is nontrivial when $k$ is odd. Note that the explicit definition of $\left(\mathbb{Z}_{6}\right)_{\mathrm{C}+\mathrm{B}}$ in Eq. (16) is not unique due to the semidirect product in $H_{d d}$, so one can also take, e.g.,

$X=\operatorname{diag}\left(\omega^{-2}, \omega, \omega\right) \quad$ or $\quad X=\operatorname{diag}\left(\omega, \omega^{-2}, \omega\right)$.

The order parameter manifold for the symmetry breaking $G_{\mathrm{QCD}} \rightarrow H_{d d}$ by $\Phi_{d d}$ is

$$
\frac{G_{\mathrm{QCD}}}{H_{d d}}=\frac{\mathrm{SU}(3)_{\mathrm{C}} \times \mathrm{U}(1)_{\mathrm{B}}}{\mathrm{SO}(3)_{\mathrm{C}} \rtimes\left(\mathbb{Z}_{6}\right)_{\mathrm{C}+\mathrm{B}}} \simeq \frac{M_{3} \times S^{1}}{\left(\mathbb{Z}_{6}\right)_{\mathrm{C}+\mathrm{B}}}
$$

with $M_{3} \equiv \mathrm{SU}(3)_{\mathrm{C}} / \mathrm{SO}(3)_{\mathrm{C}}$. The ground state allows topologically stable vortex configurations since $\pi_{1}\left(G_{\mathrm{QCD}} / H_{d d}\right)=\mathbb{Z}$. See Refs. [59,60] for homotopy groups of $M_{3}$. The broken generators of the coset space $M_{3}$ belong to 5 representation (traceless symmetric $2 \times 2$ tensor) of $\mathrm{SO}(3)_{\mathrm{C}}$.

\section{Symmetry breaking by the 2SC condensate}

Next, we turn on the $\Phi_{2 S C}$ in the presence of $\Phi_{d d}$ and address $H_{d d} \stackrel{\Phi_{2 S C}}{\longrightarrow} K_{2 \mathrm{SC}+d d}$ part in Eq. (11). We can divide states into two cases according to the real or complex nature of $\Phi_{2 S C}$. We will only focus on the case $\Phi_{2 S C} \in \mathbb{R}^{3}$ in this paper; The other possibility $\Phi_{2 S C} \in \mathbb{C}^{3}$, which also shows interesting physics, will be discussed in the forthcoming paper. In the case of $\Phi_{2 S C} \in \mathbb{R}^{3},\left(\Phi_{2 S C}\right)^{\alpha}$ can be transformed to one component by the unbroken gauge symmetry $\mathrm{SO}(3)_{\mathrm{C}}$ in $H_{d d}$ in Eq. (15), so that without loss of generality we can write

$$
\left(\Phi_{2 \mathrm{SC}}\right)^{\alpha}=\Delta_{2 \mathrm{SC}} \delta^{\alpha 3} .
$$

Let us consider the $\left(\mathbb{Z}_{6}\right)_{\mathrm{C}+\mathrm{B}}$ action on $\Phi_{2 \mathrm{SC}}$ in Eq. (10).

$$
\begin{aligned}
\Phi_{2 \mathrm{SC}} & \rightarrow \omega^{-2} X^{-1} \Phi_{2 \mathrm{SC}} \\
& =\left\{\begin{array}{l}
\omega^{-2} \operatorname{diag}\left(\omega, \omega, \omega^{-2}\right)^{-1} \Phi_{2 \mathrm{SC}}=\operatorname{diag}(-1,-1,1) \Phi_{2 \mathrm{SC}} \\
\omega^{-2} \operatorname{diag}\left(\omega, \omega^{-2}, \omega\right)^{-1} \Phi_{2 \mathrm{SC}}=\operatorname{diag}(-1,1,-1) \Phi_{2 \mathrm{SC}} \\
\omega^{-2} \operatorname{diag}\left(\omega^{-2}, \omega, \omega\right)^{-1} \Phi_{2 \mathrm{SC}}=\operatorname{diag}(1,-1,-1) \Phi_{2 \mathrm{SC}} .
\end{array}\right.
\end{aligned}
$$

Then, the direction of $X \in \mathbb{Z}_{6}$ inside $G_{\mathrm{QCD}}$, namely Eq. (17) or (20), is determined according to the value of $\Phi_{2 \mathrm{SC}}$. Now we took $\left(\Phi_{2 \mathrm{SC}}\right)^{\alpha}=\Delta_{2 \mathrm{SC}} \delta^{\alpha 3}$, so the symmetry $\left(\mathbb{Z}_{6}\right)_{\mathrm{C}+\mathrm{B}}$ in Eq. (23) remains unbroken because of the first line in Eq. (23). Eventually, the nonzero component in $\Phi_{2 S C}$ fixes where the $\omega^{-2}$ appears in the $X \in \mathbb{Z}_{6}$ transformation (16) with Eq. (17). An $\mathrm{SO}(2)_{\mathrm{C}}$ group remains unbroken, and the semidirect product is reduced to the direct product with the unbroken $\mathrm{SO}(2)_{\mathrm{C}}$ group:

$$
H_{d d} \rightarrow K_{2 \mathrm{SC}+d d}=\mathrm{SO}(2)_{\mathrm{C}} \times\left(\mathbb{Z}_{6}\right)_{\mathrm{C}+\mathrm{B}} .
$$

Thus, the order parameter manifold for this symmetry breaking is

$$
\frac{H_{d d}}{K_{2 \mathrm{SC}+d d}}=\frac{\mathrm{SO}(3)_{\mathrm{C}} \rtimes\left(\mathbb{Z}_{6}\right)_{\mathrm{C}+\mathrm{B}}}{\mathrm{SO}(2)_{\mathrm{C}} \times\left(\mathbb{Z}_{6}\right)_{\mathrm{C}+\mathrm{B}}} \simeq \frac{\mathrm{SO}(3)_{\mathrm{C}}}{\mathrm{SO}(2)_{\mathrm{C}}} \simeq S^{2} .
$$

\section{E. The opposite ordering}

We turn to the opposite ordering of the condensate formations given in Eq. (12). For the $\Phi_{2 S C}$ it is standard to fix the gauge as $\left(\Phi_{2 \mathrm{SC}}\right)^{\alpha}=\Delta_{2 \mathrm{SC}} \delta^{\alpha 3}$, and it is kept invariant under the symmetry 


$$
\tilde{H}_{2 \mathrm{SC}}=\mathrm{SU}(2)_{\mathrm{C}} \times \mathrm{U}(1)_{\mathrm{C}+\mathrm{B}} .
$$

The order parameter manifold of this breaking is

$$
\frac{G_{\mathrm{QCD}}}{\tilde{H}_{2 \mathrm{SC}}}=\frac{\mathrm{SU}(3)_{\mathrm{C}}}{\mathrm{SU}(2)_{\mathrm{C}}}
$$

allowing trivial first homotopy group or no stable vortices.

Now we turn on $\Phi_{d d}$. The upper-left block of $\Phi_{d d}$ can be diagonalized by the $\mathrm{SU}(2)_{\mathrm{C}}$ transformation in $\tilde{H}_{2 \mathrm{SC}}$ :

$$
\Phi_{d d}=\left(\begin{array}{ccc}
\Delta_{d d} & 0 & \left(\Phi_{d d}\right)_{13} \\
0 & \Delta_{d d}^{\prime} & \left(\Phi_{d d}\right)_{23} \\
\left(\Phi_{d d}\right)_{13} & \left(\Phi_{d d}\right)_{23} & \Delta_{d d}^{\prime \prime}
\end{array}\right)
$$

Here, as before, we have assumed the diagonal components to be equal. To identify an unbroken symmetry, we see that the $\mathbb{Z}_{6}$ action in Eq. (16) with $X$ in Eq. (17) acts on $\Phi_{d d}$ as

$$
\begin{aligned}
\Phi_{d d} & \rightarrow \omega^{-2} X \Phi_{d d} X^{T} \\
& =\left(\begin{array}{ccc}
\Delta_{d d} & 0 & \omega^{3}\left(\Phi_{d d}\right)_{13} \\
0 & \Delta_{d d}^{\prime} & \omega^{3}\left(\Phi_{d d}\right)_{23} \\
\omega^{3}\left(\Phi_{d d}\right)_{13} & \omega^{3}\left(\Phi_{d d}\right)_{23} & \Delta_{d d}^{\prime \prime}
\end{array}\right) .
\end{aligned}
$$

Then, there are two phases depending on the absence or presence of the off-diagonal components. When the offdiagonal components vanish, the unbroken group is $K=$ $K_{2 \mathrm{SC}+d d} \simeq \mathrm{SO}(2)_{\mathrm{C}} \times\left(\mathbb{Z}_{6}\right)_{\mathrm{C}+\mathrm{B}}$ as the same as Eq. (24). The case that the off-diagonal components are present corresponds to the case that $\Phi_{2 S C} \in \mathbb{C}^{3}$ above Eq. (22) for the symmetry breaking in Eq. (11). This case will be mentioned in the forthcoming paper.

The order parameter manifolds for these breakings are

$$
\frac{\tilde{H}_{2 \mathrm{SC}}}{K_{2 \mathrm{SC}+d d}}=\frac{\mathrm{SU}(2)_{\mathrm{C}}}{\mathrm{SO}(2)_{\mathrm{C}}} \times \frac{\mathrm{U}(1)_{\mathrm{C}+\mathrm{B}}}{\left(\mathbb{Z}_{6}\right)_{\mathrm{C}+\mathrm{B}}} \simeq S^{2} \times \frac{\mathrm{U}(1)_{\mathrm{C}+\mathrm{B}}}{\left(\mathbb{Z}_{6}\right)_{\mathrm{C}+\mathrm{B}}} .
$$

Topologically stable vortex configurations appears in the ground state according to $\pi_{1}\left(\tilde{H}_{2 \mathrm{SC}} / K_{2 \mathrm{SC}+d d}\right)=\mathbb{Z}$.

\section{MINIMAL TOPOLOGICAL VORTEX IN $\langle d d\rangle$ PHASE-NON-ABELIAN ALICE STRING}

In this section, we examine the vortices that appear in the presence of $\Phi_{d d}$. As introduced in Sec. III C, we discuss that the topologically stable minimal configuration is a nonAbelian vortex rather than a superfluid vortex (Sec. III A) or a color-magnetic flux tube (Sec. III B) similar to the CFL case. We also discuss that our non-Abelian vortices which show the property akin to that of Alice string, namely, the topological obstruction.

\section{A. Abelian vortex}

The simplest vortex is a superfluid vortex of the form

$$
\Phi_{d d}(\varphi)=f_{0}(r) e^{i \varphi} \Delta_{d d} \mathbf{1}_{3} \sim e^{i \varphi} \Delta_{d d} \mathbf{1}_{3}
$$

with the boundary condition $f_{0}(0)=0$ and $f_{0}(\infty)=1$ for the profile function $f$, and $(r, \varphi)$ being the polar coordinates. This carries a unit quantized circulation in $\mathrm{U}(1)_{\mathrm{B}}$ as encoded in the factor $e^{i \varphi}$. We call this a $\mathrm{U}(1)_{\mathrm{B}}$ superfluid vortex or an Abelian vortex. See the first line of Table I. This string is created under rotation because of the superfluidity. Although this string is topologically stable due to $\pi_{1}\left[\mathrm{U}(1)_{\mathrm{B}}\right]=\mathbb{Z}$, it is unstable against decay into three nonAbelian Alice strings, as discussed below in Sec. IV C.

\section{B. Pure color flux tubes}

A color-magnetic flux tube generated by a closed loop in the group manifold $\mathrm{SU}(3)_{\mathrm{C}}$ is given (see Refs. $[25,61]$ for the case of the CFL and 2SC phases, respectively)

$$
\begin{aligned}
\Phi_{d d}(\varphi) & =\Delta_{d d}\left(\begin{array}{ccc}
f(r) e^{-2 i \varphi} & 0 & 0 \\
0 & f(r) e^{-2 i \varphi} & 0 \\
0 & 0 & f(r) e^{4 i \varphi}
\end{array}\right), \\
A_{i} & =-\frac{a(r)}{g} \frac{\epsilon_{i j} x^{j}}{r^{2}} \operatorname{diag}(-1,-1,2),
\end{aligned}
$$

where $g$ is the $\mathrm{SU}(3)$ gauge coupling constant and the boundary conditions for the profile functions $f$ and $a$ are set by

TABLE I. AB phases of light $(u, d)$ quarks, heavy $(s)$ quark, and the 2 SC condensate $\Phi_{2 S C} \sim u d$ around various vortices. For row vectors, their columns represent the colors of the quarks or $\Phi_{2 S C}$. For $3 \times 3$ matrices, rows represent the colors of fluxes of the vortices and columns represent the colors of the quarks or $\Phi_{2 S C}$. The order $k$ of the $s$-quark AB phase $\mathbb{Z}_{k}$ corresponds to the flux $1 / k$ of the vortex.

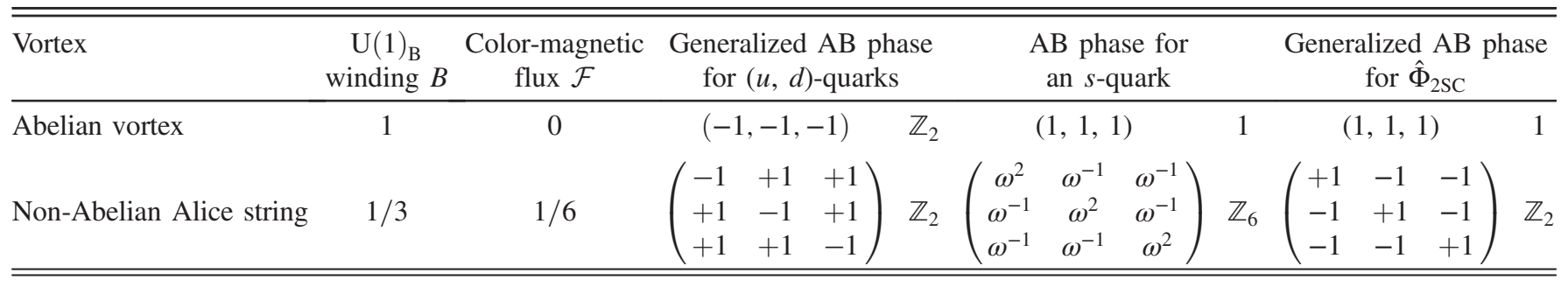




$$
f(0)=a(0)=0, \quad f(\infty)=a(\infty)=1 .
$$

Here, we have set the condensate at $\varphi=0$ and large distance as

$$
\Phi_{d d}(\varphi=0)=\Delta_{d d} f(r) \mathbf{1}_{3} \sim \Delta_{d d} \mathbf{1}_{3} .
$$

This carries a color-magnetic flux

$$
\int d^{2} x F_{12}=\frac{2 \pi}{g} \operatorname{diag}(-1,-1,2)=\mathcal{F}_{0} \operatorname{diag}(-1,-1,2),
$$

where $F_{12}$ is the color-magnetic component of the field strength tensor and we have defined a unit color-magnetic flux $\mathcal{F}_{0}$ as

$$
\mathcal{F}_{0} \equiv \frac{2 \pi}{g}
$$

This flux tube is unstable and thus decay into the ground state because of $\pi_{1}\left[\mathrm{SU}(3)_{\mathrm{C}}\right]=0$. We will not discuss the color flux tubes henceforth, and only focus on the topologically stable objects.

\section{Non-Abelian Alice strings}

Here, we present the most stable vortex in this system, that is a vortex with orientational moduli in the internal non-Abelian gauge space, which we call as a non-Abelian Alice string. This string can be characterized by the holonomy at infinite distance

$$
U(\varphi)=\mathcal{P} \exp \left(i g \int_{0}^{\varphi} \boldsymbol{A} \cdot d \ell\right) .
$$

This generates the condensate winding at spatial infinity:

$$
\Phi_{d d}(\varphi)=e^{i \varphi / 3} U(\varphi) \Phi_{d d}(\varphi=0) U^{T}(\varphi) .
$$

The ansatz that we impose here is of the form of

$$
\begin{gathered}
\Phi_{d d}(\varphi)=\Delta_{d d}\left(\begin{array}{ccc}
g(r) & 0 & 0 \\
0 & g(r) & 0 \\
0 & 0 & f(r) e^{i \varphi}
\end{array}\right), \\
A_{i}=-\frac{a(r)}{6 g} \frac{\epsilon_{i j} x^{j}}{r^{2}} \operatorname{diag}(-1,-1,2),
\end{gathered}
$$

where the corresponding holonomy (37) is given by

$$
U(\varphi)=e^{i(\varphi / 6) \operatorname{diag}(-1,-1,2)},
$$

and we have set the condensate at $\varphi=0$ and large distance as
$\Phi_{d d}(\varphi=0)=\Delta_{d d}\left(\begin{array}{ccc}g(r) & 0 & 0 \\ 0 & g(r) & 0 \\ 0 & 0 & f(r)\end{array}\right) \sim \Delta_{d d} \mathbf{1}_{3}$.

The boundary conditions for the profiles $f$ and $g$ are

$$
f(0)=g^{\prime}(0)=a(0)=0, \quad f(\infty)=g(\infty)=a(\infty)=1 .
$$

The form of the condensate and the gauge field in Eq. (39) can be understood as follows. Coupling between the condensate and the gauge field comes from the minimal coupling in the covariant derivative $D_{\mu} \Phi=\partial_{\mu} \Phi-i g A_{\mu} \Phi$. The profile of the vortex can be made such that it only appear in $T_{8} \propto \operatorname{diag}(-1,-1,2)$ component of the gauge field with suitable gauge transformation while keeping the kinetic term $\left|D_{\mu} \Phi\right|^{2}$ in the energy minimized. One can factor out the winding in $\mathrm{U}(1)_{\mathrm{B}}$ in Eq. (39) to make the color-magnetic flux clearer

$\Phi_{d d}(\varphi)=\Delta_{d d} e^{i \varphi / 3}\left(\begin{array}{ccc}g(r) e^{-i \varphi / 3} & 0 & 0 \\ 0 & g(r) e^{-i \varphi / 3} & 0 \\ 0 & 0 & f(r) e^{2 i \varphi / 3}\end{array}\right)$.

From this expression it is evident that this carries $1 / 6$ quantized color-magnetic flux, $\mathcal{F}=\mathcal{F}_{0} / 6$ (see Eq. (36) for the definition of $\mathcal{F}_{0}$ ), and $1 / 3$ quantized circulation in $\mathrm{U}(1)_{\mathrm{B}}$, as summarized in the second line of Table I. Since $\Phi_{d d}$ ought to be single valued, $U(2 \pi)$ belongs to the little group $H_{d d}$ of the condensate $\Phi_{d d}(0)$. Therefore, this configuration indeed connects two elements of $\mathrm{SU}(3)_{\mathrm{C}}: U(\varphi=0)=\mathbf{1}_{3}$ and $U(\varphi=2 \pi)=$ $e^{i(\pi / 3) \operatorname{diag}(-1,-1,2)}=\operatorname{diag}\left(\omega^{-1}, \omega^{-1}, \omega^{2}\right)$. Note that the latter does not belong to the center of $\mathrm{SU}(3)_{\mathrm{C}}$.

The configuration in Eq. (39) is not the unique. Two other typical configurations are given by

$$
\begin{aligned}
\Phi_{d d}(\varphi) & =\Delta_{d d}\left(\begin{array}{ccc}
g(r) & 0 & 0 \\
0 & f(r) e^{i \varphi} & 0 \\
0 & 0 & g(r)
\end{array}\right), \\
U(\varphi) & =e^{i(\varphi / 6) \operatorname{diag}(-1,2,-1)}, \\
A_{i} & =-\frac{a(r)}{6 g} \frac{\epsilon_{i j} x^{j}}{r^{2}} \operatorname{diag}(-1,2,-1),
\end{aligned}
$$

and 


$$
\begin{aligned}
\Phi_{d d}(\varphi) & =\Delta_{d d}\left(\begin{array}{ccc}
f(r) e^{i \varphi} & 0 & 0 \\
0 & g(r) & 0 \\
0 & 0 & g(r)
\end{array}\right), \\
U(\varphi) & =e^{i(\varphi / 6) \operatorname{diag}(2,-1,-1)}, \\
A_{i} & =-\frac{a(r)}{6 g} \frac{\epsilon_{i j} x^{j}}{r^{2}} \operatorname{diag}(2,-1,-1) .
\end{aligned}
$$

We denote configurations in Eqs. (39), (44), and (45) by fluxes that they carry, that is, $b, g$, and $r$, respectively.

These three configurations are just typical configurations among a more general continuous family of configurations

$$
\begin{aligned}
\Phi_{d d}(\varphi) & =e^{i \varphi / 3} U^{O}(\varphi) \Phi_{d d}(0) U^{O T}(\varphi) \\
& =\Delta_{d d} O\left(\begin{array}{ccc}
g(r) & 0 & 0 \\
0 & g(r) & 0 \\
0 & 0 & f(r) e^{i \varphi}
\end{array}\right) O^{T}, \\
U^{O}(\varphi) & =O e^{i(\varphi / 6) \operatorname{diag}(-1,-1,2)} O^{T} \\
& =e^{i(\varphi / 6) O \operatorname{diag}(-1,-1,2) O^{T}}, \\
A_{i} & =-\frac{a(r)}{6 g} \frac{\epsilon_{i j} x^{j}}{r^{2}} O \operatorname{diag}(-1,-1,2) O^{T},
\end{aligned}
$$

with $O \in \mathrm{SO}(3)_{\mathrm{C}}$. This continuous degeneracy can be interpreted as zero modes as follows. At spatial infinity, the residual symmetry $H=\mathrm{SO}(3) \rtimes \mathbb{Z}_{6}$ of the vacuum in the $d d$ phase should be respected. Around the core of a nonAbelian vortex, however, the symmetry is further broken spontaneously down to

$$
\tilde{K}_{\text {vortex }}=\mathrm{O}(2)_{\mathrm{C}} \times\left(\mathbb{Z}_{6}\right)_{\mathrm{C}+\mathrm{B}}
$$

by the ansatz in Eq. (39) where $f \neq g$ around the core. The symmetry $\mathrm{O}(2)_{C}$ can be decomposed as

$$
\mathrm{O}(2)_{\mathrm{C}}=\mathbb{Z}_{2} \ltimes \mathrm{SO}(2)_{\mathrm{C}},
$$

where the $\mathrm{SO}(2)_{\mathrm{C}}$ is given by

$$
\left(\begin{array}{ccc}
\cos \alpha & -\sin \alpha & 0 \\
\sin \alpha & \cos \alpha & 0 \\
0 & 0 & 1
\end{array}\right) \in \mathrm{SO}(2)_{\mathrm{C}}
$$

and $\mathbb{Z}_{2}$ is generated by $\operatorname{diag}(1,-1,-1), \operatorname{diag}(-1,1,-1)$ or their linear combinations for the configuration in Eq. (39). Note that $\left(\mathbb{Z}_{6}\right)_{\mathrm{C}+\mathrm{B}}$ symmetry remains unbroken because its action given in Eq. (16) is represented with the block diagonal matrix of the form:

$$
X^{k}=\left(\begin{array}{cc|c}
\omega^{k} & 0 & 0 \\
0 & \omega^{k} & 0 \\
\hline 0 & 0 & \omega^{-2 k}
\end{array}\right) .
$$

Consequently, there appear Nambu-Goldstone (NG) modes associated with this spontaneous symmetry breaking in the vicinity of the vortex:

$$
O \in \frac{H_{0}}{\tilde{K}_{\text {vortex }}}=\frac{\mathrm{SO}(3)_{\mathrm{C}} \rtimes \mathbb{Z}_{6}}{\mathrm{O}(2)_{\mathrm{C}} \times \mathbb{Z}_{6}} \simeq S^{2} / \mathbb{Z}_{2} \simeq \mathbb{R} P^{2} .
$$

In the vortex core, there remains the unbroken gauge symmetry [62].

\section{PROPERTIES OF NON-ABELIAN ALICE STRINGS}

In this section, we study some characteristic properties of non-Abelian Alice strings, namely topological obstruction, $\mathrm{AB}$ phases, and decay of a $\mathrm{U}(1)_{\mathrm{B}}$ vortex into three Alice strings.

\section{A. Topological obstruction of a non-Abelian Alice string}

In this subsection, we will turn to the properties of nonAbelian Alice string. In the presence of the string, the unbroken little group $H_{\varphi}$ is position (in this case the angle $\varphi$ ) dependent. These little groups are related by the gauge transformation $U(\varphi)$ given in Eq. (37)

$$
H_{\varphi}=U(\varphi) H_{\varphi=0} U^{-1}(\varphi)
$$

and all of them are isomorphic to the one at $\varphi=0$, $H_{\varphi=0}=H_{d d}=\mathrm{SO}(3) \times \mathbb{Z}_{6}$. Therefore, for whole group $H_{\varphi=2 \pi} \simeq H_{\varphi=0}$. However, it is not true for an individual generator of $H_{\varphi}[41,42,44]$. The fact that the embedding of $H_{\varphi}$ in $G_{\mathrm{QCD}}$ is position dependent prevents the global and continuous definition of the generators of $H_{\varphi}$ for all values of the angle $\varphi$.

Consider the generators of $\mathrm{SO}(3), T_{x}, T_{y}$ and $T_{z}$. Let us define

$$
T_{x, y, z}(\varphi) \equiv U(\varphi) T_{x, y, z} U^{-1}(\varphi)
$$

then we can show

$$
\begin{gathered}
T_{x, y}(\varphi=2 \pi)=-T_{x, y} \neq T_{x, y}(\varphi=0) \\
T_{z}(\varphi=2 \pi)=+T_{z}=T_{z}(\varphi=0)
\end{gathered}
$$

for non-Abelian Alice string with the flux of the color $b$ in Eq. (39). We can only recover the original $T_{x, y}$ by rotating $\varphi=4 \pi$ : 


$$
T_{x, y}(\varphi=4 \pi)=T_{x, y}(\varphi=0) .
$$

Likewise, similar nonsingle-valuedness are present around Alice strings with different fluxes:

$$
\begin{gathered}
T_{z, x}(\varphi=2 \pi)=-T_{z, x} \neq T_{z, x}(\varphi=0) \\
T_{y}(\varphi=2 \pi)=+T_{y}=T_{y}(\varphi=0)
\end{gathered}
$$

for non-Abelian Alice string with the flux of the color $g$ in Eq. (44), and

$$
\begin{gathered}
T_{y, z}(\varphi=2 \pi)=-T_{y, z} \neq T_{y, z}(\varphi=0) \\
T_{x}(\varphi=2 \pi)=+T_{x}=T_{x}(\varphi=0)
\end{gathered}
$$

for non-Abelian Alice string with the flux of the color $r$ in Eq. (45).

This phenomenon that we have shown here is the socalled topological obstruction $[41,42,44]$. One of the prominent example of the topological obstruction can be found in the theory with the string configuration dubbed an Alice string for which a U(1) generator flips its sign after complete encirclement of the string [40-42,44,48-50,63]. We have shown here that the vortex that exists in the $\langle d d\rangle$ phase in dense QCD is in fact the non-Abelian analog of the Alice string. Hereafter, we will call this vortex configuration as a non-Abelian Alice string.

Because of the topological obstruction, one can state that the unbroken symmetry $H$ of the ground state is further "broken" to its subgroup $\tilde{K}=\mathrm{O}(2) \times \mathbb{Z}_{6}$ whose generators are all single-valued. Eventually, this breaking is the same with spontaneous breaking in the vicinity of the vortex in Eq. (47).

\section{B. Generalized Aharonov-Bohm phases around vortices}

In the case of the CFL phase, the electromagnetic $\mathrm{AB}$ phases around a non-Abelian vortex was calculated [66]. In this subsection, we consider $\mathrm{AB}$ phases of color $\mathrm{SU}(3)_{\mathrm{C}}$ symmetry around a vortex, without switching on the electromagnetism. To this end, we put the quark field $\hat{q}$, the gauge field $A_{i}$ and the 2SC diquark operator $\hat{\Phi}_{2 \mathrm{SC}}$ in the above-mentioned vortex configuration. When they go around the vortex, they undergo a gauge transformation according to the Wilson-line integral in Eq. (37). Thus, after a complete encirclement of the vortex, the fields may pick up a phase coming from the Wilson loop and the baryon circulation in $\mathrm{U}(1)_{\mathrm{B}}$. We call the phase without the baryon circulation as $\mathrm{AB}$ phase and the one with the baryon circulation as generalized $\mathrm{AB}$ phase. In the later sections, we use this (generalized) $\mathrm{AB}$ phase as a guiding principle to ensure the consistency between the different fields.

For any $\varphi \neq 0$, the quark field operator $\hat{q}$ and the diquark operator $\hat{\Phi}_{2 \mathrm{SC}}$ at $\varphi$ are given by a holonomy action as

$$
\begin{gathered}
\hat{q}(\varphi) \sim e^{i \theta_{\mathrm{B}}(\varphi)} U(\varphi) \hat{q}(\varphi=0), \\
\hat{\Phi}_{2 \mathrm{SC}}(\varphi) \sim e^{2 i \theta_{\mathrm{B}}(\varphi)} U^{-1}(\varphi) \hat{\Phi}_{2 \mathrm{SC}}(\varphi=0),
\end{gathered}
$$

where $U(\varphi)$ is defined as in Eq. (37). One can read out the generalized $\mathrm{AB}$ phase (in the exponentiated form) $\Gamma$ from the fields at $\varphi=2 \pi$ after encircling around the vortex, i.e., $\hat{q}(0) \rightarrow \hat{q}(2 \pi)=\Gamma \hat{q}(0)$ and $\hat{\Phi}_{2 \mathrm{SC}}(0) \rightarrow \hat{\Phi}_{2 \mathrm{SC}}(2 \pi)=$ $\Gamma \hat{\Phi}_{2 \mathrm{SC}}(0)$. As discussed above, our gauge field $A_{i}$ is proportional to the diagonal matrix. So, taking $A_{i} \propto$ $\operatorname{diag}(-1,-1,2)$ for instance, we can explicitly write down

$$
\begin{aligned}
\hat{q}(2 \pi) & \sim e^{i \theta_{\mathrm{B}}(\varphi)} U(2 \pi) \hat{q}(0) \\
& \sim e^{i \pi B} e^{2 i \pi \mathcal{F} \operatorname{diag}(-1,-1,2)}\left(\begin{array}{c}
\hat{q}_{r}(0) \\
\hat{q}_{g}(0) \\
\hat{q}_{b}(0)
\end{array}\right), \\
\hat{\Phi}_{2 \mathrm{SC}}(2 \pi) & \sim e^{2 i \theta_{\mathrm{B}}(\varphi)} U^{-1}(2 \pi) \hat{\Phi}_{2 \mathrm{SC}}(0) \\
& \sim e^{2 i \pi B} e^{-2 i \pi \mathcal{F} \operatorname{diag}(-1,-1,2)}\left(\begin{array}{c}
\hat{\Phi}_{2 \mathrm{SC}}^{r}(0) \\
\hat{\Phi}_{2 \mathrm{SC}}^{g}(0) \\
\hat{\Phi}_{2 \mathrm{SC}}^{b}(0)
\end{array}\right)
\end{aligned}
$$

where $B$ and $\mathcal{F}$ denote the $\mathrm{U}(1)_{\mathrm{B}}$ circulation and the colormagnetic flux, respectively. The values of $B$ and $\mathcal{F}$ for each vortex are tabulated in Table I. When an $s$-quark that does not participate in the condensations encircles a vortex, it receives only an $\mathrm{AB}$ phase of the color gauge group. In contrast, the light quarks $u$ and $d$, that participate in the condensations, and $\hat{\Phi}_{2 S C}$ receive an additional contribution from the baryon number symmetry $\mathrm{U}(1)_{\mathrm{B}}$ other than the usual $\mathrm{AB}$ phase of the color gauge group, because $\hat{\Phi}_{2 \mathrm{SC}}$ itself contains a vortex winding. The total phase is a generalized $\mathrm{AB}$ phase.

\section{Abelian string}

Substituting $B=1$ and $\mathcal{F}=0$ in Eq. (60), one gets the following $\mathrm{AB}$ phases. The generalized $\mathrm{AB}$ phases $\Gamma$ of light $(u, d)$ or heavy $(s)$ quarks encircling around an Abelian $\mathrm{U}(1)_{\mathrm{B}}$ vortex can be summarized, by using short-hand notation, as

$$
\begin{gathered}
\left.\Gamma_{\alpha \beta}^{u, d}(\varphi)=\begin{array}{ccc}
r & g & b \\
\left(e^{+i \varphi / 2}\right. & e^{+i \varphi / 2} & e^{+i \varphi / 2}
\end{array}\right) \\
\Gamma_{\alpha \beta}^{s}(\varphi)=\left(\begin{array}{lll}
1 & 1 & 1
\end{array}\right),
\end{gathered}
$$

where the columns $(\beta=r, g, b)$ denote the colors of the light $(u, d)$ or heavy $(s)$ quarks encircling the vortex as explicitly shown in Eq. (61). After the complete encirclement $\varphi=2 \pi$, these phases become

$$
\Gamma_{\alpha \beta}^{u, d}(\varphi=2 \pi)=\left(\begin{array}{lll}
-1 & -1 & -1
\end{array}\right),
$$




$$
\Gamma_{\alpha \beta}^{s}(\varphi=2 \pi)=\left(\begin{array}{lll}
+1 & +1 & +1
\end{array}\right) .
$$

On the other hand, when the $2 \mathrm{SC}$ condensate $\Phi_{2 \mathrm{SC}}$ encircles the vortex, its $\mathrm{AB}$ phases are

$$
\Gamma_{\alpha \beta}^{2 \mathrm{SC}}(\varphi)=\left(\begin{array}{lll}
e^{+i \varphi} & e^{+i \varphi} & e^{+i \varphi}
\end{array}\right) .
$$

After the complete encirclement $\varphi=2 \pi$, these phases become

$$
\Gamma_{\alpha \beta}^{2 \mathrm{SC}}(\varphi=2 \pi)=\left(\begin{array}{lll}
+1 & +1 & +1
\end{array}\right) .
$$

\section{Non-Abelian Alice string}

Substituting $B=1 / 3$ and $\mathcal{F}=1 / 6$ in Eq. (60), one gets the followings. The asymptotic gauge fields of a color flux with a color $r, g, b$ are given by $A_{i}^{r} \propto \operatorname{diag}(2,-1,-1)$, $A_{i}^{g} \propto \operatorname{diag}(-1,-2,-1), A_{i}^{b} \propto \operatorname{diag}(-1,-1,2)$, respectively.

Therefore, the pure $\mathrm{AB}$ phases of heavy $(s)$ quark encircling around flux tubes can be summarized, again by using short-hand notation, as

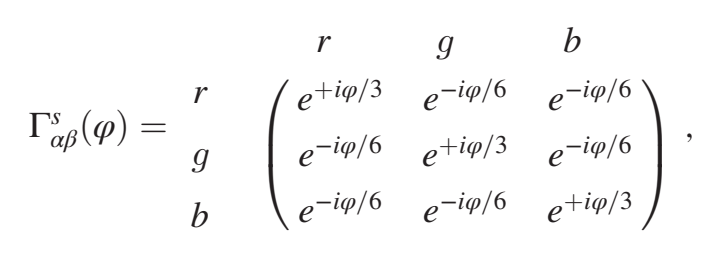

where, as explicitly indicated above, the row $(\alpha=r, g, b)$ denotes the color of the flux tubes, and the column $(\beta=r$, $g, b)$ denotes the colors of the heavy $(s)$ quark encircling them. After the complete encirclement $\varphi=2 \pi$, these phases become

$$
\Gamma_{\alpha \beta}^{s}(\varphi=2 \pi)=\left(\begin{array}{ccc}
\omega^{2} & \omega^{-1} & \omega^{-1} \\
\omega^{-1} & \omega^{2} & \omega^{-1} \\
\omega^{-1} & \omega^{-1} & \omega^{2}
\end{array}\right),
$$

which are a color nonsinglet.

When the light quarks $u, d$ encircle the Alice string, they also receive $\mathrm{U}(1)_{\mathrm{B}}$ transformation $e^{+i \varphi / 6}$ as well as the $\mathrm{AB}$ phase that they have in common with those of the $s$-quarks. Therefore, generalized $\mathrm{AB}$ phases of the light quarks $u, d$ are given by

$$
\begin{aligned}
\Gamma_{\alpha \beta}^{u, d}(\varphi) & =e^{+i \varphi / 6} \Gamma_{\alpha \beta}^{s}(\varphi) \\
& =\left(\begin{array}{ccc}
e^{+i \varphi / 2} & 1 & 1 \\
1 & e^{+i \varphi / 2} & 1 \\
1 & 1 & e^{+i \varphi / 2}
\end{array}\right)
\end{aligned}
$$

After the complete encirclement $\varphi=2 \pi$, these phases become

$$
\Gamma_{\alpha \beta}^{u, d}(\varphi=2 \pi)=\left(\begin{array}{lll}
-1 & +1 & +1 \\
+1 & -1 & +1 \\
+1 & +1 & -1
\end{array}\right)
$$

which are a color nonsinglet as well.

On the other hand, when the 2SC condensate $\Phi_{2 S C}$ encircles the Alice string, its generalized $\mathrm{AB}$ phases are

$$
\begin{aligned}
\Gamma_{\alpha \beta}^{2 \mathrm{SC}}(\varphi) & =e^{+i \varphi / 3} \Gamma_{\alpha \beta}^{S}(\varphi) \\
& =\left(\begin{array}{ccc}
1 & e^{+i \varphi / 2} & e^{+i \varphi / 2} \\
e^{+i \varphi / 2} & 1 & e^{+i \varphi / 2} \\
e^{+i \varphi / 2} & e^{+i \varphi / 2} & 1
\end{array}\right) .
\end{aligned}
$$

After the complete encirclement $\varphi=2 \pi$, these phases become

$$
\Gamma_{\alpha \beta}^{2 \mathrm{SC}}(\varphi=2 \pi)=\left(\begin{array}{ccc}
+1 & -1 & -1 \\
-1 & +1 & -1 \\
-1 & -1 & +1
\end{array}\right),
$$

which are a color nonsinglet.

The (generalized) $\mathrm{AB}$ phase of the $u, d, s$ quarks and the 2SC condensate $\Phi_{2 S C}$ are different among the colors, so they are color nonsinglet. Surprisingly, by winding the quarks or the $2 \mathrm{SC}$ condensate around the string, one can read out the color of the flux from infinite distance [67].

\section{Decay of an Abelian $\mathrm{U}(\mathbf{1})_{B}$ vortex}

Here we discuss that a single Abelian $\mathrm{U}(1)_{\mathrm{B}}$ superfluid vortex decays into a set of three non-Abelian Alice strings with total color magnetic flux canceled out, as the case of the CFL phase $[10,20,51,52]$.

Superfluid vortices have logarithmically divergent tension (energy per unit length), $E \sim n^{2} \log \Lambda$, in a system of the size $\Lambda$. Here, $n$ is the winding number of $\mathrm{U}(1)_{\mathrm{B}}$, or the superfluid circulation. An Abelian $\mathrm{U}(1)_{\mathrm{B}}$ vortex in Eq. (31) has $n=1$ while a non-Abelian Alice string in Eq. (39), (44), (45) or more generally Eq. (46) has $n=1 / 3$. Thus, this fact implies a decay of Abelian vortices into a set of three non-Abelian vortices as schematically shown in Fig. 3(a), because of energetics

$$
E(n=1)=9 E(n=1 / 3)>3 E(n=1 / 3) .
$$

If three non-Abelian vortices are infinitely separated, the total energy is proportional to $3 E(n=1 / 3)$, which is $1 / 3$ of the energy of a single Abelian vortex.

The $\mathrm{U}(1)_{\mathrm{B}}$ vortex has no color-magnetic flux while nonAbelian Alice strings in Eq. (39), (44), (45) do with their sum canceled out. We thus conclude

$$
\Phi_{d d}=\Phi_{d d}^{\mathrm{U}(1)_{\mathrm{B}}} \rightarrow \Phi_{d d}=\Phi_{d d}^{r}+\Phi_{d d}^{g}+\Phi_{d d}^{b},
$$




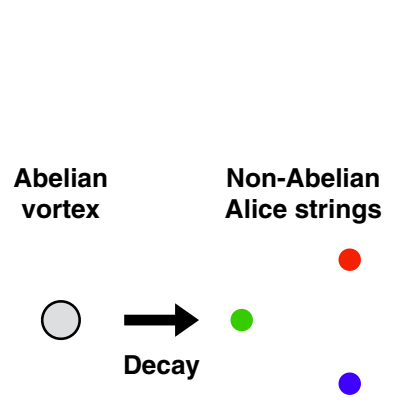

(a)

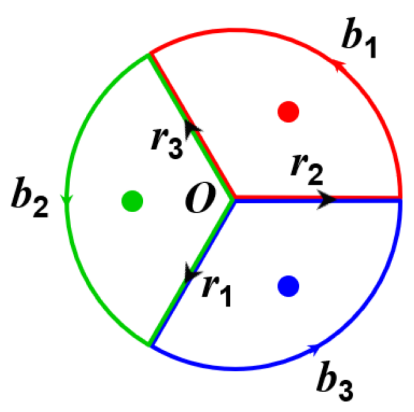

(b)
FIG. 3. Detailed configurations of decays of a $U(1)_{B}$ superfluid vortex. (a) Schematic illustration of the decay of an Abelian vortex into three non-Abelian Alice strings. (b) The configurations after decay. The $\mathrm{U}(1)_{\mathrm{B}}$ vortex initially located at the origin $O$ decays into three non-Abelian Alice strings, denoted by the red, green, blue blobs. The $b_{1}, b_{2}$, and $b_{3}$ are the paths with angles $2 \pi / 3$ at the boundary at the spatial infinity, and $r_{1}, r_{2}, r_{3}$ denote the paths from the origin $O$ to spatial infinities.

$$
A_{i}=A_{i}^{\mathrm{U}(1)_{\mathrm{B}}}=0 \rightarrow A_{i}=A_{i}^{r}+A_{i}^{g}+A_{j}^{b}
$$

with

$$
\begin{aligned}
\Phi_{d d}^{\mathrm{U}(1)_{\mathrm{B}}} & =\Delta_{d d} \operatorname{diag}\left(f_{0}(r) e^{i \varphi}, f_{0}(r) e^{i \varphi}, f_{0}(r) e^{i \varphi}\right), \\
\Phi_{d d}^{r} & =\Delta_{d d} \operatorname{diag}\left(g(r), g(r), f(r) e^{i \varphi}\right), \\
\Phi_{d d}^{g} & =\Delta_{d d} \operatorname{diag}\left(g(r), f(r) e^{i \varphi}, g(r)\right), \\
\Phi_{d d}^{b} & =\Delta_{d d} \operatorname{diag}\left(f(r) e^{i \varphi}, g(r), g(r)\right), \\
A_{i}^{r} & =-\frac{a^{r}(r)}{6 g} \frac{\epsilon_{i j} x_{1}^{j}}{r_{1}^{2}} \operatorname{diag}(-1,-1,2), \\
A_{i}^{g} & =-\frac{a^{g}(r)}{6 g} \frac{\epsilon_{i j} x_{2}^{j}}{r_{2}^{2}} \operatorname{diag}(-1,2,-1), \\
A_{i}^{b} & =-\frac{a^{b}(r)}{6 g} \frac{\epsilon_{i j} x_{3}^{j}}{r_{3}^{2}} \operatorname{diag}(2,-1,-1) .
\end{aligned}
$$

Let us check the consistency in terms of the generalized $\mathrm{AB}$ phases and topological obstructions. To this end, we divide a closed loop at infinity encircling the $\mathrm{U}(1)_{\mathrm{B}}$ vortex to $b_{1}, b_{2}$, and $b_{3}$ as in Fig. 3(b). Along the paths $b_{1}, b_{2}$, and $b_{3}$, the $\mathrm{U}(1)_{\mathrm{B}}$ symmetry acts as $\exp (i \varphi / 3)$ giving rise to a $\omega^{2}$ factor so that each path carries $1 / 3$ circulation and the whole loop $b_{1}+b_{2}+b_{3}$ carries the unit circulation of the $\mathrm{U}(1)_{\mathrm{B}}$ vortex. We define radial paths $r_{1}, r_{2}$ and $r_{3}$ starting at the origin to infinities as in Fig. 3. The closed loop $l_{r}=b_{1}-r_{3}+r_{2}, l_{g}=b_{2}-r_{1}+r_{3}$, and $l_{b}=b_{3}-r_{2}+$ $r_{1}$ enclose $r, g$ and $b$ non-Abelian Alice strings, respectively.

Then we find that the $\mathrm{SU}(3)_{\mathrm{C}}$ gauge transformations along the radial paths $r_{1}, r_{2}$, and $r_{3}$ are given by

$$
\begin{aligned}
& r_{1}: U=\exp (i F(r) / 6) \operatorname{diag}(1,-1,0), \\
& r_{2}: U=\exp (i F(r) / 6) \operatorname{diag}(-1,0,1), \\
& r_{3}: U=\exp (i F(r) / 6) \operatorname{diag}(0,1,-1),
\end{aligned}
$$

respectively, with an arbitrary function $F(r)$ satisfying the boundary conditions

$$
F(r=0)=0, \quad F(r=\infty)=2 \pi .
$$

Then, the loops $l_{r}, l_{g}$, and $l_{b}$ enclosing $r, g$, and $b$ nonAbelian Alice strings correctly recover the original group actions of the following elements $\left(e^{i \varphi / 3}, U\right) \in \mathrm{U}(1)_{\mathrm{B}} \times$ $S U(3)_{C}$ :

$l_{r}=b_{1}-r_{3}+r_{2}:\left(e^{i \varphi / 3}, U=e^{i F / 6} \operatorname{diag}(-1,-1,2)\right)$,

$l_{g}=b_{2}-r_{1}+r_{3}:\left(e^{i \varphi / 3}, U=e^{i F / 6} \operatorname{diag}(-1,2,-1)\right)$,

$l_{b}=b_{3}-r_{2}+r_{1}:\left(e^{i \varphi / 3}, U=e^{i F / 6} \operatorname{diag}(2,-1,-1)\right)$,

respectively.

When we encircle three non-Abelian strings $(r, g, b)$, total $\mathrm{AB}$ phases of any particles coincide with those of a $\mathrm{U}(1)_{\mathrm{B}}$ vortex, as can be seen from Table I. For $u, d$ quarks, if one multiply all three elements of each column (color of $u, d$ quarks) of the third line of Table I, one get $(+1)(+1)(-1)=$ -1 for all colors of $u, d$ quarks, which coincides with the first line for a $\mathrm{U}(1)_{\mathrm{B}}$ vortex. The same holds for a $s$ quark $\omega^{2} \omega^{-1} \omega^{-1}=1$ for all colors of the $s$ quark.

Furthermore, although each non-Abelian string exhibits topological obstruction given in Eq. (54), (56), or (57), the total topological obstruction is cancelled out when we encircle all the three non-Abelian strings $(r, g, b)$. Thus, a topological obstruction is dynamically created under the decay of a $\mathrm{U}(1)_{\mathrm{B}}$ string.

\section{TURNING ON THE 2SC CONDENSATE, AND BULK-SOLITON MODULI LOCKING}

So far we have seen the vortex configurations in the presence of the $\langle d d\rangle$ condensate $\Phi_{d d}$. In particular, there appear the non-Abelian vortices, which can be regarded as a non-Abelian extension of the so-called Alice string. Here, in this section, we will turn on the $2 \mathrm{SC}$ condensate $\Phi_{2 \mathrm{SC}}$ in this vortex background, and show that the $2 \mathrm{SC}$ condensate $\Phi_{2 S C}$ will be aligned toward the direction of the flux.

\section{A. Single non-Abelian Alice string}

As mentioned in Sec. II D, one can use the remaining gauge degrees of freedom in the presence of $\Phi_{d d}$ to transform $\Phi_{2 S C}$ into one component. When turning on $\Phi_{2 S C}$, therefore we can take ansatze of the form $\Phi_{2 S C}=$ $\left(\Delta_{2 \mathrm{SC}}, 0,0\right)^{T},\left(0, \Delta_{2 \mathrm{SC}}, 0\right)^{T}$ or $\left(0,0, \Delta_{2 \mathrm{SC}}\right)^{T}$. We will have to consider their gauge rotation consistently with the $\langle d d\rangle$ condensate $\Phi_{d d}$. In the presence of a single non-Abelian 
Alice string of Eq. (39), $\Phi_{2 S C}$ receives generalized $\mathrm{AB}$ phases as (see Sec. IV B)

$$
\begin{aligned}
\Phi_{2 \mathrm{SC}} & \rightarrow e^{i \varphi / 3} U^{-1}(\varphi) \Phi_{2 \mathrm{SC}} \\
& =\left(\begin{array}{ccc}
e^{i \varphi / 2} & 0 & 0 \\
0 & e^{i \varphi / 2} & 0 \\
0 & 0 & 1
\end{array}\right) \Phi_{2 \mathrm{SC}} \\
& =\left\{\begin{array}{l}
\left(e^{i \varphi / 2} \Delta_{2 \mathrm{SC}}, 0,0\right)^{T} \\
\left(0, e^{i \varphi / 2} \Delta_{2 \mathrm{SC}}, 0\right)^{T} \\
\left(0,0, \Delta_{2 \mathrm{SC}}\right)^{T}
\end{array}\right.
\end{aligned}
$$

for $\Phi_{2 \mathrm{SC}}=\left(\Delta_{2 \mathrm{SC}}, 0,0\right)^{T},\left(0, \Delta_{2 \mathrm{SC}}, 0\right)^{T}$ or $\left(0,0, \Delta_{2 \mathrm{SC}}\right)^{T}$, respectively. This explicitly shows that at $\varphi=2 \pi$, if the first or the second component of $\Phi_{2 S C}$ is nonzero, they would not be single-valued, to be inconsistent. Therefore, it cannot be realized. One is only left with the possibility where the third component of $\Phi_{2 S C}$ is nonzero, i.e., $\Phi_{2 \mathrm{SC}}=\left(0,0, \Delta_{2 \mathrm{SC}}\right)^{T}$, and it implies that in the presence of the non-Abelian vortex of $d d$ diquark condensate, the 2SC condensate must be aligned along the direction of the non-Abelian moduli from the consistency.

This alignment of two condensates should be compared with the zero mode appearing in the vicinity of the vortex, the Alice string moduli $\mathbb{R} P^{2}$ in Eq. (51). Apparently, in the presence of an Alice string in Eq. (44) or (45), $\Phi_{2 S C}$ must be $\Phi_{2 \mathrm{SC}}=\left(0, \Delta_{2 \mathrm{SC}}, 0\right)^{T}$ or $\Phi_{2 \mathrm{SC}}=\left(\Delta_{2 \mathrm{SC}}, 0,0\right)^{T}$, respectively.

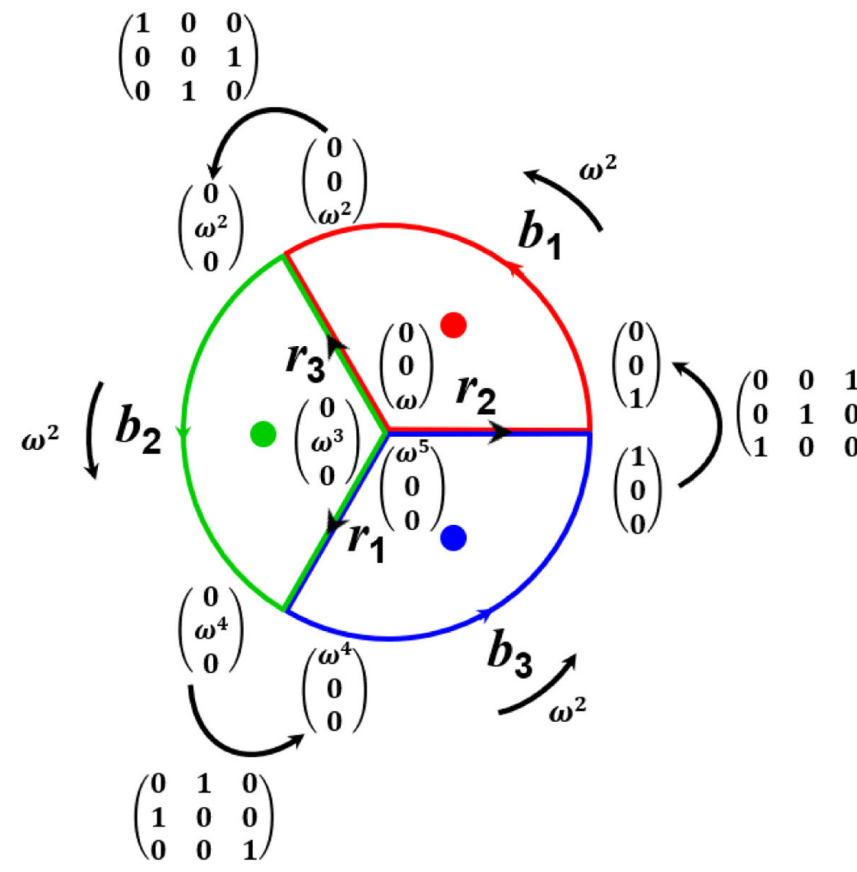

(a)
Thus, the expectation value of the $2 \mathrm{SC}$ condensate $\Phi_{2 S C}$ is aligned to the direction of color-magnetic flux or the moduli of the Alice string. We call this phenomenon as a "bulksoliton moduli locking". Conversely, since the 2SC condensate $\Phi_{2 S C}$ is the bulk quantity, it may be natural to say that rather the Alice string moduli $\mathbb{R} P^{2}$ are locked to $\Phi_{2 S C}$. In this picture, the Alice string is "Abelianized," and if there are several Alice strings, all Alice string moduli are aligned to $\Phi_{2 S C}$.

\section{B. Decay of an Abelian $\mathrm{U}(\mathbf{1})_{\mathrm{B}}$ vortex}

One may wonder whether the $2 \mathrm{SC}$ condensate $\Phi_{2 \mathrm{SC}}$ might prevent the decay of a $\mathrm{U}(1)_{\mathrm{B}}$ superfluid vortex into three non-Abelian Alice strings (see discussion in Sec. IV $\mathrm{C}$ ), since it is a nontrivial question whether the $2 \mathrm{SC}$ condensate $\Phi_{2 S C}$ can be locked to each non-Abelian vortex consistently to cover the entire space. In this subsection, we show that the decay of a $\mathrm{U}(1)_{\mathrm{B}}$ vortex is still possible in the presence of $\Phi_{2 S C}$.

Let us discuss the configuration of $\Phi_{2 S C}$ in the configuration of the $\mathrm{U}(1)_{\mathrm{B}}$ vortex decay in Fig. 3. To this end, it is convenient to take a gauge of $\Phi_{2 S C}$ for the initial $\mathrm{U}(1)_{\mathrm{B}}$ vortex as

$$
\begin{aligned}
\Phi_{d d} & \sim e^{i \varphi} \Delta_{d d} \mathbf{1}_{3}, \\
\Phi_{2 \mathrm{SC}} & \sim \frac{\Delta_{2 \mathrm{SC}}}{\sqrt{3}} e^{i \varphi}(111)^{T} .
\end{aligned}
$$


Then, the configuration of $\Phi_{2 S C}$ after decay is almost fixed by the gauge transformation in Eq. (77) along the radial paths $r_{1}, r_{2}$, and $r_{3}$, and $\mathrm{U}(1)_{\mathrm{B}}$ transformation $\omega^{2}$ along the boundary paths $b_{1}, b_{2}$, and $b_{3}$ at infinity. Another condition is the absence of $\mathrm{AB}$ defects around the non-Abelian Alice strings, fixing nonzero components of $\Phi_{2 S C}$ around each non-Abelian Alice string due to the bulk-soliton moduli locking. We thus obtain a possible configuration of $\Phi_{2 S C}$ as summarized in Fig. 4(a). Note that while the phase of $\Phi_{2 S C}$ varies along the radial paths $r_{1}, r_{2}$, and $r_{3}$, and the boundary paths $b_{1}, b_{2}$, and $b_{3}$ at infinity, the overall phase rotation along a closed loop encircling each non-Abelian string is cancelled out. In order to glue the boundaries between two neighboring Alice strings, one needs gauge transformations to change the position of the nonzero component of $\Phi_{2 S C}$. The remaining nontrivial point appears around the origin as zoomed up in Fig. 4(b). This can be smoothly connected to $\Phi_{2 \mathrm{SC}}^{\alpha}=\frac{\Delta_{2 S C}}{\sqrt{3}}(111)^{T}$ at the origin because of the triviality of $\pi_{1}$ for the symmetry breaking by $\Phi_{2 \mathrm{SC}}: \pi_{1}\left(G_{\mathrm{QCD}} / \tilde{H}_{2 \mathrm{SC}}\right)=0$.

We thus conclude that the $2 \mathrm{SC}$ condensate $\Phi_{2 \mathrm{SC}}$ does not prevent the decay of a $\mathrm{U}(1)_{\mathrm{B}}$ superfluid vortex into three non-Abelian Alice strings.

\section{CONSISTENCY WITH THE OPPOSITE ORDERING IN THE SYMMETRY BREAKING}

Now we address vortices appearing in the opposite ordering of the condensate formations given in Eq. (12). Namely, we now consider the case where the 2SC condensate $\Phi_{2 S C}$ forms first, followed by the $\langle d d\rangle$ condensate $\Phi_{d d}$ formation. The vortices appear corresponding to the $\Phi_{d d}$ formation because of the relation $\pi_{1}\left(\tilde{H}_{2 \mathrm{SC}} / K_{2 \mathrm{SC}+d d}\right)=\mathbb{Z}$ as already exemplified in Sec. IIE. The $2 \mathrm{SC}$ condensate in the vacuum reads $\left(\Phi_{2 \mathrm{SC}}\right)^{\alpha}=\Delta_{2 \mathrm{SC}} \delta^{\alpha 3}$.

\section{A. Superfluid vortex}

The simplest vortex is a superfluid vortex of the form

$$
\begin{gathered}
\Phi_{d d}(\varphi)=f_{0}(r) e^{i \varphi} \Delta_{d d} \mathbf{1}_{3}, \\
\Phi_{2 \mathrm{SC}}(\varphi)=h_{0}(r) e^{i \varphi}\left(00 \Delta_{2 \mathrm{SC}}\right)^{T}
\end{gathered}
$$

with the boundary conditions

$$
f_{0}(0)=h_{0}(0)=0, \quad f_{0}(\infty)=h_{0}(\infty)=1 .
$$

This arises corresponding to the breaking of $\mathrm{U}(1)_{\mathrm{B}}$ symmetry, and is exactly a $\mathrm{U}(1)_{\mathrm{B}}$ vortex considered in Sec. III A.

\section{B. Semi-superfluid $U(\mathbf{1})_{C+B}$ vortices}

Here, we consider the vortex with orientational moduli in the internal non-Abelian gauge space. The ansatz we impose here is of several kinds and we show the asymptotic form.

In the presence of the $2 \mathrm{SC}$ condensate, the residual symmetry is $\tilde{H}_{2 \mathrm{SC}}=\mathrm{SU}(2)_{\mathrm{C}} \times \mathrm{U}(1)_{\mathrm{C}+\mathrm{B}}$ as already given in Eq. (26). The $\mathrm{U}(1)_{\mathrm{C}+\mathrm{B}}$ symmetry in $\tilde{H}_{2 \mathrm{SC}}:\left\{\left(e^{i \alpha T_{8}}, e^{2 i \alpha}\right) \in\right.$ $\left.\mathrm{SU}(3)_{\mathrm{C}} \times \mathrm{U}(1)_{\mathrm{B}}: T_{8}=\operatorname{diag}(1,1,-2)\right\}$ acts on $\Phi_{d d}$ as

$$
\begin{aligned}
\Phi_{d d} & =\left(\begin{array}{ccc}
\Delta_{d d} & 0 & \left(\Phi_{d d}\right)_{13} \\
0 & \Delta_{d d}^{\prime} & \left(\Phi_{d d}\right)_{23} \\
\left(\Phi_{d d}\right)_{31} & \left(\Phi_{d d}\right)_{32} & \Delta_{d d}^{\prime \prime}
\end{array}\right) \\
& \rightarrow e^{2 i \alpha} e^{i \alpha T_{8}} \Phi_{d d}\left(e^{i \alpha T_{8}}\right)^{T} \\
& =\left(\begin{array}{ccc}
\Delta_{d d} & 0 & e^{3 i \alpha}\left(\Phi_{d d}\right)_{13} \\
0 & \Delta_{d d}^{\prime} & e^{3 i \alpha}\left(\Phi_{d d}\right)_{23} \\
e^{3 i \alpha}\left(\Phi_{d d}\right)_{31} & e^{3 i \alpha}\left(\Phi_{d d}\right)_{32} & e^{6 i \alpha} \Delta_{d d}^{\prime \prime}
\end{array}\right),
\end{aligned}
$$

while the 2SC condensate $\Phi_{2 S C} \rightarrow \Phi_{2 S C}$ is kept the same. Whether the off-diagonal blocks $\left(\Phi_{d d}\right)_{13},\left(\Phi_{d d}\right)_{23}$, $\left(\Phi_{d d}\right)_{31},\left(\Phi_{d d}\right)_{32}$ exist or not is crucial for constructions of vortices. We now restrict ourselves to the case in which the off-diagonal blocks vanish as in Sec. IIE. See the forthcoming paper for the full discussion.

When the off-diagonal blocks are absent, winding only resides in the $(3,3)$ component of $\Phi_{d d}$. Thus, we take the dependence of the condensates on the angular coordinate $\varphi$ as $\varphi=6 \alpha$ in Eq. (84):

$$
\begin{aligned}
\Phi_{2 \mathrm{SC}}^{\alpha} & =\left(00 \Delta_{2 \mathrm{SC}}\right)^{T}, \\
\Phi_{d d}(\varphi) & =e^{i \varphi / 3} U(\varphi) \Phi_{d d}(0) U^{T}(\varphi) \\
& =\Delta_{d d}\left(\begin{array}{ccc}
g(r) & 0 & 0 \\
0 & g(r) & 0 \\
0 & 0 & f(r) e^{i \varphi}
\end{array}\right), \\
U(\varphi) & =e^{i(\varphi / 6) \operatorname{diag}(-1,-1,2)} \\
A_{i} & =-\frac{a(r)}{6 g} \frac{\epsilon_{i j} x^{j}}{r^{2}} \operatorname{diag}(-1,-1,2)
\end{aligned}
$$

with the boundary conditions

$$
f(0)=g^{\prime}(0)=0, \quad f(\infty)=g(\infty)=1,
$$

where we have set the condensate at $\varphi=0$ as

$\Phi_{d d}(\varphi=0)=\Phi_{d d}\left(\begin{array}{ccc}g(r) & 0 & 0 \\ 0 & g(r) & 0 \\ 0 & 0 & f(r)\end{array}\right) \sim \Phi_{d d} \mathbf{1}_{3}$.

This carries $1 / 6$ quantized color-magnetic flux $\mathcal{F}_{0}$ and $1 / 3$ quantized circulation in $\mathrm{U}(1)_{\mathrm{B}}$. This is precisely a nonAbelian Alice string locked with $\Phi_{2 S C}$.

For each of the above-mentioned vortices other typical configurations are given by the ones in Eqs. (39), (44), and 
(45) by $r, g$, and $b$, respectively. Likewise, these three configurations can be rotated in the color space.

\section{SUMMARY AND DISCUSSIONS}

In this work we have proposed the novel topological solitons, non-Abelian Alice strings, that emerge in twoflavor dense QCD. First, we have given a brief review of the new color-superconducting phase of two-flavor dense quark matter, $2 \mathrm{SC}+\langle d d\rangle$ phase, based on the quarkhadron continuity scenario in connection with the current neutron star phenomenology [26]. There appears a color anti-symmetric diquark condensate, which is the so-called 2SC condensate [See Eq. (7)], and a color symmetric diquark condensate, which is the $\langle d d\rangle$ diquark condensate [See Eq. (8)]. Then, we have examined the residual symmetries in the presence of these diquark condensates. The result is concisely summarized in Fig. 2. We have turned on these diquark condensates in sequence, firstly the $\langle d d\rangle$ condensate, and then the 2SC condensate, and have identified the unbroken subgroups [See the right side of Fig. 2] as well as the corresponding order parameter manifolds. The new feature here is that the unbroken subgroup $H_{d d}$ has the semi-direct product, which leads to the Alice phenomenon. We have also done the consistency check with the opposite ordering of turning on the diquark condensates [See the left side of Fig. 2].

Based on these symmetry breaking patterns, we have introduced the vortices appearing in the presence of $\langle d d\rangle$ diquark. The central object in this paper is the non-Abelian Alice string introduced in Sec. III C. This carries the fractional winding in $\mathrm{U}(1)_{\mathrm{B}}$ and also a color-magnetic flux. We have set forth the characteristic property of this string, i.e., topological obstruction. We have introduced the notion of the generalized $\mathrm{AB}$ phase, which is the combination of the $\mathrm{U}(1)_{\mathrm{B}}$ circulation and the gauge rotation around the vortex. Along these lines, we have discussed the decay of an Abelian $\mathrm{U}(1)_{\mathrm{B}}$ vortex. The Abelian $\mathrm{U}(1)_{\mathrm{B}}$ vortex is the simplest object in the superfluid matter, however, it is not the most stable one. We have shown on the basis of the energetics and the generalized $A B$ phase that a single Abelian vortex should be separated into a triple of the non-Abelian Alice strings.

We have only considered the existence of $\langle d d\rangle$ up to here, but when turning on the 2SC diquark, then one must take care of the consistency in terms of the gauge transformation. Again the guiding principle is the generalized $\mathrm{AB}$ phase. We have explicitly shown that in the presence of $\langle d d\rangle$ the form of the 2SC ansatz is restricted and should be aligned toward the winding component of the $\langle d d\rangle$ condensate. Then we have been lead to the observation that the soliton moduli, which are the degrees of freedom corresponding to the winding component of the vortex [See also Eq. (51)], are actually locked with the bulk quantity, which is nothing but the $2 \mathrm{SC}$ diquark here. We named this phenomenon as a "bulk-soliton moduli locking". We have also checked that in the presence of the $2 \mathrm{SC}$ condensate, the decay of a $\mathrm{U}(1)_{\mathrm{B}}$ vortex is still possible.

Finally, we have assessed the case when we turned around the ordering of the diquark formations in order to maintain the consistency. The vortices in this case is the same as the ones discussed above. This is as we have expected because we will finally arrive at the identical coexistence phase of the $2 \mathrm{SC}$ and $\langle d d\rangle$, so the physics should not depend on this ordering.

In this paper, we have restricted ourselves to the most symmetric form of the ansatze for the condensates. In particular, we have considered the case of $\Phi_{2 S C} \in \mathbb{R}^{3}$ in this paper; The other possibility $\Phi_{2 S C} \in \mathbb{C}^{3}$ will be discussed elsewhere. We have also assumed that eigenvalues of $\langle d d\rangle$ are degenerate. Basically, they do not have to be degenerate, and we can consider more general setup. Another simplification was the absence of the electromagnetism. For the CFL phase, effects of turning on the electromagnetism on vortices can be incorporated as the effective potential on the $\mathbb{C} P^{2}$ moduli space [68]. Such an effect on the $\mathbb{R} P^{2}$ moduli space could be studied for the case of the $2 \mathrm{SC}+\langle d d\rangle$ phase as well.

The major motivation to consider the $2 \mathrm{SC}+\langle d d\rangle$ phase is the quark-hadron continuity between two-flavor quark and hadronic matter. Thus, it is a natural question how vortices in the $2 \mathrm{SC}+\langle d d\rangle$ phase discussed in this paper are connected to those in the ${ }^{3} P_{2}$ hadronic phase. In the ${ }^{3} P_{2}$ phase, there are half-quantized vortices [69] in addition to integer vortices [70-74]. It was also shown in Ref. [37] that ${ }^{3} P_{2}$ superfluids are topological superfluids exhibiting topologically protected gapless Majorana fermions on their boundary [37] or inside vortex cores [74]. Thus, it is an interesting question whether such gapless fermion modes exist in vortex cores in $2 \mathrm{SC}+\langle d d\rangle$ phase to study validity of quark-hadron continuity. As this problem concerns, gapless Majorana fermion modes indeed exist in non-Abelian vortices in the CFL phase $[75,76]$. Also, in the higher density region, the $2 \mathrm{SC}+$ $\langle d d\rangle$ phase may be connected to the CFL phase. Thus, it is also an intriguing question how vortices in the $2 \mathrm{SC}+$ $\langle d d\rangle$ phase are connected to those in the CFL phase. The strange quark mass will take a crucial part in studying along this line, and the effect of quark masses has already been discussed for non-Abelian vortices in the CFL phase [54]. These and related topics are to be explored in future studies.

It is often the case that when quasiparticles exhibit nontrivial $\mathrm{AB}$ phases around a vortex, the system is topologically ordered. It was, however, discussed in Refs. [22,23] that the CFL phase is not topologically ordered albeit a non-Abelian nature of the $\mathrm{AB}$ phases of vortices. This is because of superfluidity of the CFL phase. The same would hold for $2 \mathrm{SC}+\langle d d\rangle$ phase discussed in this paper. Concerning this issue, when $\mathrm{U}(1)_{\mathrm{B}}$ is coupled to a $\mathrm{U}(1)$ gauge field, the system is topologically ordered [77]. 
Thus, the $2 \mathrm{SC}+\langle d d\rangle$ phase would give a novel topologically ordered phase if the $\mathrm{U}(1)_{\mathrm{B}}$ is gauged.

We have studied nontrivial $A B$ phases of quasiparticles around strings. In the case of non-Abelian strings, exchanging strings themselves may give rise to a nontrivial braiding statistics, as discussed in Ref. [46]. Regarding this, when Majorana fermion zero modes exist in the case of topological superconductors such as chiral $P$-wave superconductors, it also gives rise to another kind of nonAbelian braidings [78]. It is also actually the case for non-Abelian vortices in the CFL phase $[79,80]$ due to Majorana fermion zero modes $[75,76]$. These two facts suggest, in the case of the $2 \mathrm{SC}+\langle d d\rangle$ phase, a very interesting possibility of novel non-Abelian statistics having two different origins.

\section{ACKNOWLEDGMENTS}

We thank Shigehiro Yasui for a discussion at the early stage of this work. Y. F. thanks Kenji Fukushima for reading the manuscript and comments. This work is supported in part by Grant-in-Aid for Scientific Research, JSPS KAKENHI Grants No. JP20J10506 (Y.F.) and No. JP18H01217 (M. N.).
[1] M. G. Alford, A. Schmitt, K. Rajagopal, and T. Schäfer, Rev. Mod. Phys. 80, 1455 (2008).

[2] M. G. Alford, K. Rajagopal, and F. Wilczek, Nucl. Phys. B537, 443 (1999).

[3] M. G. Alford, K. Rajagopal, and F. Wilczek, Phys. Lett. B 422, 247 (1998).

[4] R. Rapp, T. Schäfer, E. V. Shuryak, and M. Velkovsky, Phys. Rev. Lett. 81, 53 (1998).

[5] M. Eto, Y. Hirono, M. Nitta, and S. Yasui, Prog. Theor. Exp. Phys. 2014, 012D01 (2014).

[6] M. M. Forbes and A. R. Zhitnitsky, Phys. Rev. D 65, 085009 (2002).

[7] K. Iida and G. Baym, Phys. Rev. D 66, 014015 (2002).

[8] C. Vafa and E. Witten, Nucl. Phys. B234, 173 (1984).

[9] A. Balachandran, S. Digal, and T. Matsuura, Phys. Rev. D 73, 074009 (2006).

[10] E. Nakano, M. Nitta, and T. Matsuura, Phys. Rev. D 78, 045002 (2008).

[11] E. Nakano, M. Nitta, and T. Matsuura, Prog. Theor. Phys. Suppl. 174, 254 (2008).

[12] M. Eto and M. Nitta, Phys. Rev. D 80, 125007 (2009).

[13] T. Schäfer and F. Wilczek, Phys. Rev. Lett. 82, 3956 (1999).

[14] M. G. Alford, J. Berges, and K. Rajagopal, Nucl. Phys. B558, 219 (1999).

[15] K. Fukushima, Phys. Rev. D 70, 094014 (2004).

[16] T. Hatsuda, M. Tachibana, N. Yamamoto, and G. Baym, Phys. Rev. Lett. 97, 122001 (2006); N. Yamamoto, M. Tachibana, T. Hatsuda, and G. Baym, Phys. Rev. D 76, 074001 (2007).

[17] T. Hatsuda, M. Tachibana, and N. Yamamoto, Phys. Rev. D 78, 011501 (2008).

[18] A. Schmitt, S. Stetina, and M. Tachibana, Phys. Rev. D 83, 045008 (2011).

[19] M. G. Alford, G. Baym, K. Fukushima, T. Hatsuda, and M. Tachibana, Phys. Rev. D 99, 036004 (2019).

[20] C. Chatterjee, M. Nitta, and S. Yasui, Phys. Rev. D 99, 034001 (2019); J. Phys. Soc. Jpn. Conf. Proc. 26, 024030 (2019).

[21] A. Cherman, S. Sen, and L. G. Yaffe, Phys. Rev. D 100, 034015 (2019).
[22] Y. Hirono and Y. Tanizaki, Phys. Rev. Lett. 122, 212001 (2019).

[23] Y. Hirono and Y. Tanizaki, J. High Energy Phys. 07 (2019) 062.

[24] A. Cherman, T. Jacobson, S. Sen, and L. G. Yaffe, arXiv:2007.08539.

[25] M. G. Alford and A. Sedrakian, J. Phys. G 37, 075202 (2010).

[26] Y. Fujimoto, K. Fukushima, and W. Weise, Phys. Rev. D 101, 094009 (2020); Y. Fujimoto, arXiv:2002.08073.

[27] E. Annala, T. Gorda, A. Kurkela, J. Nättilä, and A. Vuorinen, Nat. Phys. 16, 907 (2020).

[28] K. Masuda, T. Hatsuda, and T. Takatsuka, Astrophys. J. 764, 12 (2013); Prog. Theor. Exp. Phys. 2013, 073 D01 (2013).

[29] T. Kojo, P. D. Powell, Y. Song, and G. Baym, Phys. Rev. D 91, 045003 (2015).

[30] G. Baym, T. Hatsuda, T. Kojo, P. D. Powell, Y. Song, and T. Takatsuka, Rep. Prog. Phys. 81, 056902 (2018).

[31] P. Demorest, T. Pennucci, S. Ransom, M. Roberts, and J. Hessels, Nature (London) 467, 1081 (2010); E. Fonseca et al., Astrophys. J. 832, 167 (2016); J. Antoniadis et al., Science 340, 1233232 (2013); H. T. Cromartie et al., Nat. Astron. 4, 72 (2020).

[32] M. Hoffberg, A. Glassgold, R. Richardson, and M. Ruderman, Phys. Rev. Lett. 24, 775 (1970).

[33] R. Tamagaki, Prog. Theor. Phys. 44, 905 (1970); T. Takatsuka and R. Tamagaki, Prog. Theor. Phys. 46, 114 (1971); T. Takatsuka, Prog. Theor. Phys. 47, 1062 (1972).

[34] R. W. Richardson, Phys. Rev. D 5, 1883 (1972).

[35] J. Sauls and J. Serene, Phys. Rev. D 17, 1524 (1978).

[36] T. Takatsuka and R. Tamagaki, Prog. Theor. Phys. Suppl. 112, 27 (1993).

[37] T. Mizushima, K. Masuda, and M. Nitta, Phys. Rev. B 95, 140503 (2017).

[38] S. Yasui, C. Chatterjee, and M. Nitta, Phys. Rev. C 99, 035213 (2019).

[39] S. Yasui, C. Chatterjee, M. Kobayashi, and M. Nitta, Phys. Rev. C 100, 025204 (2019).

[40] A. S. Schwarz, Nucl. Phys. B208, 141 (1982). 
[41] M. G. Alford, K. Benson, S. R. Coleman, J. March-Russell, and F. Wilczek, Phys. Rev. Lett. 64, 1632 (1990); 65, 668(E) (1990).

[42] M. G. Alford, K. Benson, S. R. Coleman, J. March-Russell, and F. Wilczek, Nucl. Phys. B349, 414 (1991).

[43] M. G. Alford, K.-M. Lee, J. March-Russell, and J. Preskill, Nucl. Phys. B384, 251 (1992).

[44] J. Preskill and L. M. Krauss, Nucl. Phys. B341, 50 (1990).

[45] M. Bucher, H.-K. Lo, and J. Preskill, Nucl. Phys. B386, 3 (1992).

[46] H.-K. Lo and J. Preskill, Phys. Rev. D 48, 4821 (1993).

[47] U. Leonhardt and G. E. Volovik, Pis'ma Zh. Eksp. Teor. Fiz. 72, 66 (2000).

[48] C. Chatterjee and M. Nitta, J. High Energy Phys. 09 (2017) 046.

[49] C. Chatterjee and M. Nitta, Eur. Phys. J. C 77, 809 (2017).

[50] C. Chatterjee and M. Nitta, Phys. Rev. D 101, 085002 (2020).

[51] M. Cipriani, W. Vinci, and M. Nitta, Phys. Rev. D 86, 121704 (2012).

[52] M. G. Alford, S. Mallavarapu, T. Vachaspati, and A. Windisch, Phys. Rev. C 93, 045801 (2016).

[53] M. Eto, E. Nakano, and M. Nitta, Phys. Rev. D 80, 125011 (2009).

[54] M. Eto, M. Nitta, and N. Yamamoto, Phys. Rev. Lett. 104, 161601 (2010).

[55] K. Osterwalder and E. Seiler, Ann. Phys. (N.Y.) 110, 440 (1978).

[56] E. H. Fradkin and S. H. Shenker, Phys. Rev. D 19, 3682 (1979).

[57] T. Banks and E. Rabinovici, Nucl. Phys. B160, 349 (1979).

[58] It is known in the nematic phase [35] for which $\operatorname{diag}(1, r, 1-r)$ is implied for the $i, j$ indices, with real parameter $r$.

[59] R. Auzzi and M. Shifman, J. Phys. A 40, 6221 (2007).

[60] R. Auzzi, S. Bolognesi, and M. Shifman, Phys. Rev. D 77, 125029 (2008).

[61] K. Iida, Phys. Rev. D 71, 054011 (2005).

[62] This is in contrast to the case of a non-Abelian vortex in the CFL phase, accompanied by the $\mathbb{C} P^{2}$ orientational moduli $[5,10,53,54]$.
[63] In Refs. [48-50], an $\mathrm{SU}(2) \times \mathrm{U}(1)$ gauge theory with charged triplet scalar fields were studied, which is an $\mathrm{SU}(2)$ version of our case where $\mathrm{U}(1)$ is also gauged. The case of a global U(1) symmetry, closer to our case, was discussed in Refs. $[64,65]$ in the context of the axion dark matter model.

[64] R. Sato, F. Takahashi, and M. Yamada, Phys. Rev. D 98, 043535 (2018).

[65] C. Chatterjee, T. Higaki, and M. Nitta, Phys. Rev. D 101, 075026 (2020).

[66] C. Chatterjee and M. Nitta, Phys. Rev. D 93, 065050 (2016).

[67] This is in contrast to the case of non-Abelian vortices (color flux tubes) in the CFL phase, around which all (generalized) AB phases are color singlet [20].

[68] W. Vinci, M. Cipriani, and M. Nitta, Phys. Rev. D 86, 085018 (2012).

[69] K. Masuda and M. Nitta, Prog. Theor. Exp. Phys. 2020, 013D01 (2020).

[70] P. Muzikar, J. Sauls, and J. Serene, Phys. Rev. D 21, 1494 (1980).

[71] J. Sauls, D. Stein, and J. Serene, Phys. Rev. D 25, 967 (1982).

[72] K. Masuda and M. Nitta, Phys. Rev. C 93, 035804 (2016).

[73] C. Chatterjee, M. Haberichter, and M. Nitta, Phys. Rev. C 96, 055807 (2017).

[74] Y. Masaki, T. Mizushima, and M. Nitta, Phys. Rev. Research 2, 013193 (2020).

[75] S. Yasui, K. Itakura, and M. Nitta, Phys. Rev. D 81, 105003 (2010).

[76] T. Fujiwara, T. Fukui, M. Nitta, and S. Yasui, Phys. Rev. D 84, 076002 (2011).

[77] Y. Hidaka, Y. Hirono, M. Nitta, Y. Tanizaki, and R. Yokokura, Phys. Rev. D 100, 125016 (2019).

[78] D. Ivanov, Phys. Rev. Lett. 86, 268 (2001).

[79] S. Yasui, K. Itakura, and M. Nitta, Phys. Rev. B 83, 134518 (2011).

[80] Y. Hirono, S. Yasui, K. Itakura, and M. Nitta, Phys. Rev. B 86, 014508 (2012). 